\title{
Maternal Motivation: Exploring the Roles of Prolactin and Pup Stimuli
}

\author{
Hugo Salais-López ${ }^{a}$ María Abellán-Álvaro ${ }^{a}$ b María Bellés ${ }^{a} \quad$ Enrique Lanuza ${ }^{b}$ \\ Carmen Agustin-Pavon ${ }^{\mathrm{b}}$ Fernando Martínez-García ${ }^{\mathrm{a}}$ \\ aResearch Unit in Functional Neuroanatomy, Unitat Predepartamental de Medicina, Universitat Jaume I, \\ Castelló de la Plana, Spain; besearch Unit in Functional Neuroanatomy, Departament de Biologia Cellular, \\ Funcional i Antropologia Física, Universitat de València, Burjassot, Spain
}

\section{Keywords}

Amygdala $\cdot$ Behaviour - Motherhood .

Immunohistochemistry · Prolactin · Sociosexual brain network

\begin{abstract}
Motherhood entails increased motivation for pups, which become strong reinforcers and guide maternal behaviours. This depends on steroids and lactogens acting on the brain of females during pregnancy and postpartum. Since virgin female mice exposed to pups are nearly spontaneously maternal, the specific roles of endocrine and pup-derived signals in the induction of maternal motivation remain unclear. This work investigates maternal motivation in dams and virgin female mice, using a novel variant of the pup retrieval paradigm, the motivated pup retrieval test. We also analyse the role of prolactin (PRL) and of stimuli derived from a litter of pups and its mother, in the acquisition of maternal motivation. Experimental design included female mice in 3 conditions: lactating dams, comothers (virgins housed and sharing pup care with dams) and pup-naïve virgins. Females underwent 3 motivated-pup-retrieval trials, with pups displaced
\end{abstract}

karger@karger.com www.karger.com/nen

(C) 2020 S. Karger AG, Basel

Karger ${ }^{\prime}=$ behind a 10-cm-high wire-mesh barrier. Dams retrieved with significantly lower latencies than comothers or virgins, indicating that full maternal motivation appears only after pregnancy. Although initially comothers and virgins showed no retrieval, comothers significantly improved throughout the experiment, suggesting an induced sensitization process. Lengthening exposure of comothers to the dyad pups-dam (from 2 to 5 days at the beginning of testing) had no strong effects on maternal sensitization. PRL responsiveness was analysed in these animals using immunohistochemical detection of phosphorylated signal transducer and activator of transcription 5 (pSTAT5, PRL-derived signalling marker). As expected, dams showed significantly higher pSTAT5 expression in most of the analysed nuclei. Moreover, comothers displayed significantly higher PRL responsiveness than pupnaïve virgins in the medial preoptic nucleus, even if they display similar circulating PRL levels, which are significantly lower than those of dams. Given the instrumental role of this nucleus in the relay and integration of pup-derived stimuli to facilitate proactive maternal responses, this increase in $P R L$ responsiveness likely reflects the mechanism underlying the maternal sensitization process reported in this work. Since the analyses of maternal motivation and PRL signalling 
in the brain were performed in the same animals, we were able to explore correlation between both set of data. The results shed light on the neuroendocrine mechanisms underlying maternal motivation and other aspects of maternal behaviour.

(c) 2020 S. Karger AG, Basel

\section{Introduction}

Maternal behaviour includes species-specific social interactions aimed at facilitating infant survival until its reproductive maturity [1]. These behaviours also promote correct development that ensures physical and mental health of the offspring [2-4]. In rodents, which are common models for the neurobiological study of maternal behaviour, maternal behaviours are classified in pup-directed behaviours and non-pup-directed ones. The former, also known as maternal care, includes pup retrieval to the nest, crouching over the pups, nursing, and licking/ grooming them. Non-pup-directed behaviours consist of building and maintaining the nest and defending it against potentially threatening conspecifics, for example, maternal aggression [5].

Maternal behaviours can also be classified into automatic (reflexive), elicited by proximal pup stimulation and largely dependent on brainstem/spinal cord circuits (e.g., nursing), and proactive maternal responses [6], motivated search for pups and retrieval behaviours that are likely dependent on forebrain circuits. In fact, most pupdirected maternal behaviours require a high level of motivation towards pups in order to be expressed properly. In line with this, rat dams bar-press at a significantly higher rate than virgin females if rewarded with a pup they are able to interact with [7], whereas early postpartum rats significantly prefer pup-associated versus cocaine-associated environments in a conditioned placepreference task [8]. Thus, motherhood entails a specific motivational state maintained during the peripartum period, when pups and their associated stimuli acquire a strong incentive salience and elicit proactive maternal responses [9].

Maternal behaviours typically emerge around parturition, prompted by profound functional changes in the female brain. The major agents regulating these changes are endocrine signals of pregnancy (and lactation), including mainly gonadal steroids (estradiol and progesterone) together with lactogenic hormones (prolactin [PRL] and placental lactogens) [10]. These changes start during late pregnancy, thus assuring proper expression of maternal behaviours by the time of delivery, whereas during the postpartum period, pup-derived stimuli seem to trigger, guide, and maintain maternal behaviours [11].

In contrast to rats, where virgin females initially avoid pups and only display a certain degree of maternal care [12] after 6-8 days of daily exposure to pups [13], virgin female mice lack this initial avoidance of pups. In fact, using different models of pup-sensitized virgin females, our laboratory $[14]$ and others $[15,16]$ have found that virgin female mice do not differ significantly from dams when facing a conventional pup retrieval test (classical paradigm to measure maternal motivation), as they retrieve pups as quickly as lactating dams after only 1 or 2 days of experience, and many of them even retrieve pups in their first test. Although this might suggest that in mice, maternal care would be largely independent of pregnancyrelated endocrine signals, few previous studies regard maternal motivation specifically [15]. Their results suggest that some motivational aspects of maternal behaviour can be induced in virgin females in comparable levels to dams without the need of hormones (oestradiol) acting on the brain. Here, we specifically analyse the motivational component of maternal behaviour and the role of pup stimuli and PRL in its induction.

To do so, we have first designed a test for assessing maternal motivation, the motivated pup retrieval test (MPRT), in which experimental animals must perform a physically demanding task in order to successfully retrieve pups to the nest (climb a barrier back and forth; for more details, see Material and Methods section). The test is based on the fact that goal-directed tasks requiring a high effort are performed only for highly rewarding stimuli $[17,18]$. Using this MPRT, we have compared the motivation of 4 experimental groups of female mice for retrieving pups: lactating dams, short-term comothers (STCs) and long-term comothers (LTCs), and pup-naïve virgins. Lactating dams are under the influence of both pregnancy/lactation-related endocrine signals and pupderived stimuli. The comother model of maternal sensitization $[14,19]$ consists of virgin females which are cohoused with a (non-experimental) pregnant female through parturition and postpartum and thus are granted continuous interaction with pups since their birth. Comothers are thus exposed to infant- and dam-derived sensory stimulation but seemingly not to pregnancy- or lactation-related hormones. In order to evaluate the dynamics of maternal sensitization, we included 2 groups of comothers having experienced 2 days (STCs) or 5 days (LTCs) of exposure to pups prior to the MPRT. Finally, pup-naïve virgin controls lack both pup-derived and 
motherhood hormonal stimulation. The comparison of these groups allowed us to assess the relative contribution of endocrine signals and pup-derived (or dam-derived) external stimuli to the onset of maternal motivation.

In the second part of this work, we focussed on the neuroendocrine substrate of maternal sensitization. In the rat, evidence shows that this process has a clear endocrine component, since either blood transfusions from dams [20] or the administration of PRL under a proper gonadal steroid background [21], drastically shorten sensitization latency. However, the exact role of endocrine inputs on the induction of maternal motivation has not been analysed in mice yet. In this regard, we hypothesize that endocrine signals of motherhood, specifically lactogenic hormones [22], might play a significant role in maternal sensitization, paralleling its instrumental role in rats $[23,24]$. However, although this causal relationship between PRL/lactogenic action and maternal behaviour is well established, it is still unknown where in the brain this hormonal action is promoting maternal behaviours and, specifically, maternal motivation. To address this issue, we have analysed the expression of phosphorylated signal transducer and activator of transcription 5 (pSTAT5, a reliable indicator of PRL-derived signal transduction [25-27]) in specific nuclei of the sociosexual brain network of females subject to the MPRT. By comparing the central patterns of pSTAT5 expression of comothers with those of dams and virgin females (positive and negative controls, respectively), we expect to gain a deeper insight on the putative role of PRL in the regulation of maternal sensitization and maternal behaviour in female mice. A correlational analysis of the levels of pSTAT5 with the score in the MPRT might further contribute to clarify: (a) whether motivated maternal behaviour in dams and virgin females (comothers) is related to PRL action in the brain; (b) the key locations where PRL action might promote maternal motivation in dams and, eventually, in comothers. In addition, we also analyse correlation between PRL signalling (density of pSTAT5 immunoreactivity) in the different brain centres under scrutiny, to explore whether the different nuclei are responding in the same way to the circulating levels of PRL.

Finally, in an additional experiment, we checked if a hormonal change occurs in virgin females because of exposure to pups and a pregnant/lactating female (the dam), which might explain changes in motivation for pups. To do so, we compared the circulating levels of PRL in pupnaïve virgins and comothers (pup-sensitized virgin females) by means of ELISA.

Neuroendocrinology of Maternal

Motivation
The results suggest that pup-derived stimuli and maybe cohabitation with a pregnant female are able to induce maternal sensitization through a process not mediated by circulating PRL but seemingly involving changes in central PRL signalling in specific brain centres. Our results also suggest possible brain structures mediating the influence of PRL in the induction of maternal motivation for pups and in other aspects of maternal behaviour.

\section{Materials and Methods}

\section{Animals and Experimental Design}

Animals were treated throughout according to the European Union Council Directive of June 3, 2010 (6106/1/10 REV1) and procedures were approved by the Committee of Ethics on Animal Experimentation of the Jaume I University of Castellón (UJI), where the experiments were performed.

In this experiment, a total of $n=105$ female CD1 mice of 10 weeks of age were used (Janvier, France). Of these, 33 animals arrived on pregnancy day 10 to our animal facility, together with 72 virgin females. At the moment of arrival, females were pair-housed in polypropylene cages (145 mm wide, $465 \mathrm{~mm}$ long, and $215 \mathrm{~mm}$ high; Panlab) under controlled temperature $\left(24 \pm 2^{\circ} \mathrm{C}\right)$ and lighting conditions ( $12 \mathrm{~h}: 12 \mathrm{~h}$; lights $\mathrm{ON}$ at 8 a.m.), with ad libitum access to food and water.

\section{Experiment 1: Motivated Pup-Retrieval and PRL Signalling}

For the first experiment, at arrival, females were randomly distributed into 4 experimental groups (Fig. 1): lactating dams $(n=$ 10), STCs (pup-sensitized virgin females, see below, $n=10$ ), LTCs $(n=8)$, and pup-naïve virgins $(n=10)$. In order to balance the housing conditions, experimental comothers were cohoused with a non-experimental dam, experimental dams were cohoused with a non-experimental comother, and virgins were housed in pairs (Fig. 1). The morning after parturition, litters were culled down to 8 pups to ensure homogeneous interaction of the experimental animals with pups, as some aspects of maternal behaviour are influenced by litter size [28]. One of the pregnant females was removed from the experiment due to problems during labour, leaving $n=9$ lactating dams. Females underwent the MPRT daily for 3 consecutive days (Fig. 1). The next day, they were perfused and brain tissue was collected and processed for the immunohistochemical detection of pSTAT5.

Behavioural Testing: MPRT

The MPRT was a modified pup retrieval in which the experimental females had to retrieve the pups off the nest site by climbing a 10-cm-high wire-mesh barrier (Fig. 1), similar to the one used by Kohl et al. [29]. The actual height of the barrier was validated on previous pilot tests (data not shown) to provide the proper difficulty to the task. At the beginning of the test, the experimental female and her non-experimental partner were removed from their home cage, and 2 barriers were glued to the walls, one in each corner of the cage distal to the nest. Then, 4 pups were removed from the nest and 2 of them were carefully placed behind each of the barriers. The remaining 4 pups were left on the nest site, in order to prevent the experimental females from building a new nest site 

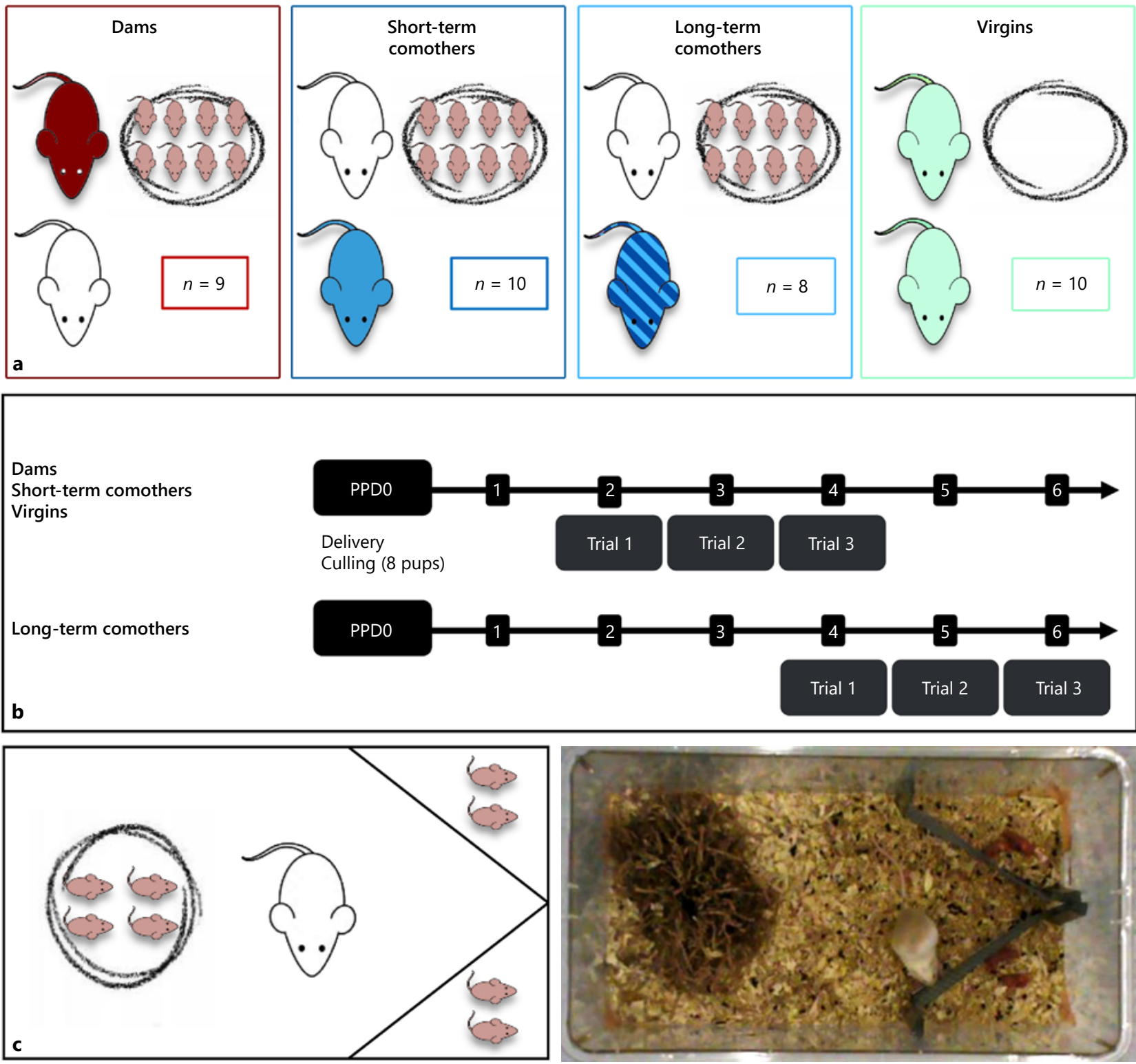

Fig. 1. Experimental Design for the MPRT. The design included 4 experimental groups of females (coloured) plus accompanying females (white, not used for the experiments), as illustrated in a dams (red, $n=9$ ), STCs (i.e., pup-sensitized virgins, blue, $n=10$ ), LTCs (striped blue, $n=8$ ), and pup-naïve virgin females (green, $n=10$ ). Dams and virgins were cohoused with an accompanying non-experimental virgin, whereas comothers were cohoused with a non-experimental pregnant female, which later gave birth and shared pup care with the comother for 2 days STCs) or 4 days (LTCs) prior to the beginning of the test. For testing, virgins and LTCs received fresh pups from donor dams. Litters were culled down to 8 pups. The test (b) consisted of 3 10-min trials, from PPD2 to PPD4 (dams, STCs, and virgins) or from PPD4 to PPD6 (LTCs). For the test (c), two 10-cm high barriers were placed in the corners of the cage distal to the nest, and 2 pups were left behind each barrier. Females (dams, STCs, and virgins) were perfused on the morning after the end of the test, and their brains processed for immunohistochemical detection of pSTAT5. STC, short-term comothers; LTCs, long-term comothers; PPD, postpartum day; MPRT, motivated pup retrieval test; pSTAT5, phosphorylated signal transducer and activator of transcription 5. 
behind either of the barriers during the test. Finally, the experimental female was placed back in the home cage and her behaviour was video-recorded for $10 \mathrm{~min}$.

Experimental dams, STCs, and virgins underwent three 10-min trials in the morning of postpartum days (PPD) 2, PPD3, and PPD4 (Fig. 1). LTCs were tested in the MPRT on days PPD4-PPD6 of their companion dams. To avoid a bias on the test due to the higher weight of pups, these comothers were given fresh pups of donor mothers in each of the experiment trials, of ages matching the pups used for STCs, dams, and virgins (ages 2, 3, and 4 days).

On the morning of PPD5, dams, STCs and pup-naïve virgins received an overdose of sodium pentobarbital (Vetoquinol, Madrid, Spain) and were perfused transcardially with $4 \%$ paraformaldehyde in $0.1 \mathrm{M}$ phosphate buffer (PB), pH 7.4 for histology. Since short-term and LTCs displayed similar behaviours (see Results), only the brains of STCs were processed and LTCs were not sacrificed.

\section{Histological Procedure}

After perfusion, brains of dams, STCs, and pup-naïve virgins were carefully extracted, post-fixed overnight in the same fixative, and cryoprotected in $30 \%$ sucrose in $0.01 \mathrm{M} \mathrm{PB}$ under gentle agitation until sinking (2-3 days). Then, the brains were cut using a freezing microtome (Microm HM-450; Walldorf, Germany) in 4 parallel series of $40-\mu \mathrm{m}$-thick coronal sections and stored thereafter in PB-30\% sucrose at $-20^{\circ} \mathrm{C}$.

A series of these coronal sections was processed for the immunohistochemical detection of pSTAT5, which constitutes a key element of PRL signalling. Immunohistochemistry was conducted as in previous works $[30,31]$. Free-floating sections underwent an initial antigen retrieval step ( 2 sequential 6 min incubations in 0.01 $\mathrm{M}$ TRIS buffer (TB), $\mathrm{pH} 10$ at $85^{\circ} \mathrm{C}$ ) and were then incubated in: (a) $1 \%$ hydrogen peroxide $\left(\mathrm{H}_{2} \mathrm{O}_{2}\right)$ for $30 \mathrm{~min}$, for endogenous peroxidase inhibition; (b) $2 \%$ BSA, $2 \%$ goat serum, and $0.3 \%$ Triton $\mathrm{X}-100$ in TBS for $1 \mathrm{~h}$, in order to block unspecific labelling; (c) rabbit anti-pSTAT5 primary antibody (pSTAT5 Tyr694; Cell Signalling Technology, Beverly, MA, USA) diluted 1:500 in TBS plus Triton X-100 $0.1 \%$ for $72 \mathrm{~h}$ at $4^{\circ} \mathrm{C}$; (d) biotinylated goat anti-rabbit IgG (Vector Laboratories, Peterborough, UK) 1:200 in TBS for 90 min; and (e) avidin-biotin-peroxidase complex (ABC Elite kit; Vector Laboratories) in TBS for $90 \mathrm{~min}$. The resulting peroxidase label was developed using 0.005\% 3-3'-diaminobenzidine (Sigma) and $0.01 \% \mathrm{H}_{2} \mathrm{O}_{2}$ in $\mathrm{TB}, \mathrm{pH} 7.6$, for about $15 \mathrm{~min}$, obtaining thereby a brown nuclear staining. Sections were rinsed in TB and mounted onto gelatinized slides, dehydrated in alcohols, cleared with xylene, and coverslipped with Entellan.

\section{Image Acquisition and Quantitative Analysis of pSTAT5}

Immunoreactivity

We assessed the density of cells showing pSTAT5 immunoreactivity (pSTAT5-ir) in a selection of brain nuclei involved in maternal behaviour expression or relevant for sociosexual behaviour regulation, where PSTAT5-ir had been described previously in virgin and lactating females [30, 32]: the lateral septum (ventral part, LSV), the medial amygdala (posterodorsal division, MePD), the central amygdala (medial division, CeM), the medial division of the bed nucleus of the stria terminalis (posteromedial part, BSTMPM), the anteroventral periventricular nucleus (AVPe), the medial preoptic nucleus (MPO), the region located between the nucleus of the anterior commissure and anterodorsal preoptic nucle- us $(\mathrm{AC} / \mathrm{ADP})$, the paraventricular hypothalamic nucleus $(\mathrm{Pa})$, the supraoptic nucleus (SO), the arcuate nucleus (Arc), the posterior intralaminar thalamic nucleus (PIL), and the lateral periaqueductal grey (LPAG). To do so, we selected representative frames of the chosen nuclei using the stereotaxic atlas of Paxinos and Franklin [33], at the anteroposterior levels (relative to Bregma) indicated in Figure 2. Thus, we obtained photomicrographs of these frames in both hemispheres (in a single section corresponding to the Bregma level indicated) using an optical microscope Leica DM 750 attached to a Leica DFC 450C digital camera (Leica AG, Germany). To do so, we adjusted gamma $=1$ and light intensity/exposure just high enough as to avoid white saturation in void areas of the preparation. Image processing and analysis was conducted on Image J software (NIH). Briefly, we subtracted background light and converted the RGB colour image to greyscale by selecting the green channel. Then, we binarized the greyscale image setting the threshold at $75 \%$ of the mode of the grey histogram, thus including every pixel below this threshold as positively labelled. We filtered smaller noise particles by an additional processing consisting of the following Image J commands: "fill holes"; "open" (3 iterations) and "watershed." Particles were additionally filtered by area (discarding those smaller than $35 \mu^{2}$, corresponding to an approximate diameter of $6.6 \mu \mathrm{m}$ ) and finally counted automatically. With this figure we calculated the mean (interhemispheric) density of pSTAT5-immunoreactive cell nuclei for each specimen by dividing the total number of particles by the total area of all the frames analysed.

\section{Experiment 2: PRL Circulating Levels in Dams, Virgins, and} Comothers

Since data from experiment 1 suggest a certain differential PRL signalling in some nuclei between pup-naïve virgin females and comothers, we decided to compare their circulating PRL levels. We used dams (PPD5) as positive controls for enhanced circulating levels and pup-naïve virgins as control group $(n=7)$. We obtained blood from groups of comothers, co-housed with pregnant females since postconception day 9-10. These comothers were sampled at dams' postconception days $14(n=6)$ and $18(n=6)$, or postpartum days PPD1 $(n=4)$, PPD5 $(n=7)$, and PPD9 $(n=7)$. This allowed us to check whether interaction with the dam during pregnancy, and/or with the dam and pups after parturition, resulted in PRL hypophyseal release in comothers (as reported for males of some mammalian species [34]).

PRL is released from the pituitary gland within a few minutes after the animals experiment a stressing stimulus [35] and i.p. administration of pentobarbital is known to alter systemic PRL levels [36]. Therefore, we avoided collecting blood samples of the experimental females of Experiment 1, after euthanasic injection during perfusion (see below). Instead, sampled animals were sacrificed by cervical dislocation and trunk blood collected, allowed to clot for $30 \mathrm{~min}$ and left at $4^{\circ} \mathrm{C}$ temperature. Then, serum was separated from the clot by centrifugation for $10 \mathrm{~min}$ at 2,000 rpm at $4^{\circ} \mathrm{C}$ and stored subsequently at $-80^{\circ} \mathrm{C}$. To assess serum PRL levels of dams, comothers and virgins, we performed a commercially available Sandwich ELISA assay for PRL (AbCam \#ab100736). All samples and standards were run simultaneously in duplicate. Absorbance values were determined at $450 \mathrm{~nm}$ using a micro-plate reader (Thermo Multiskan FC). Serum PRL concentrations were interpolated from the standard curve following a 4-point logistic regression. 

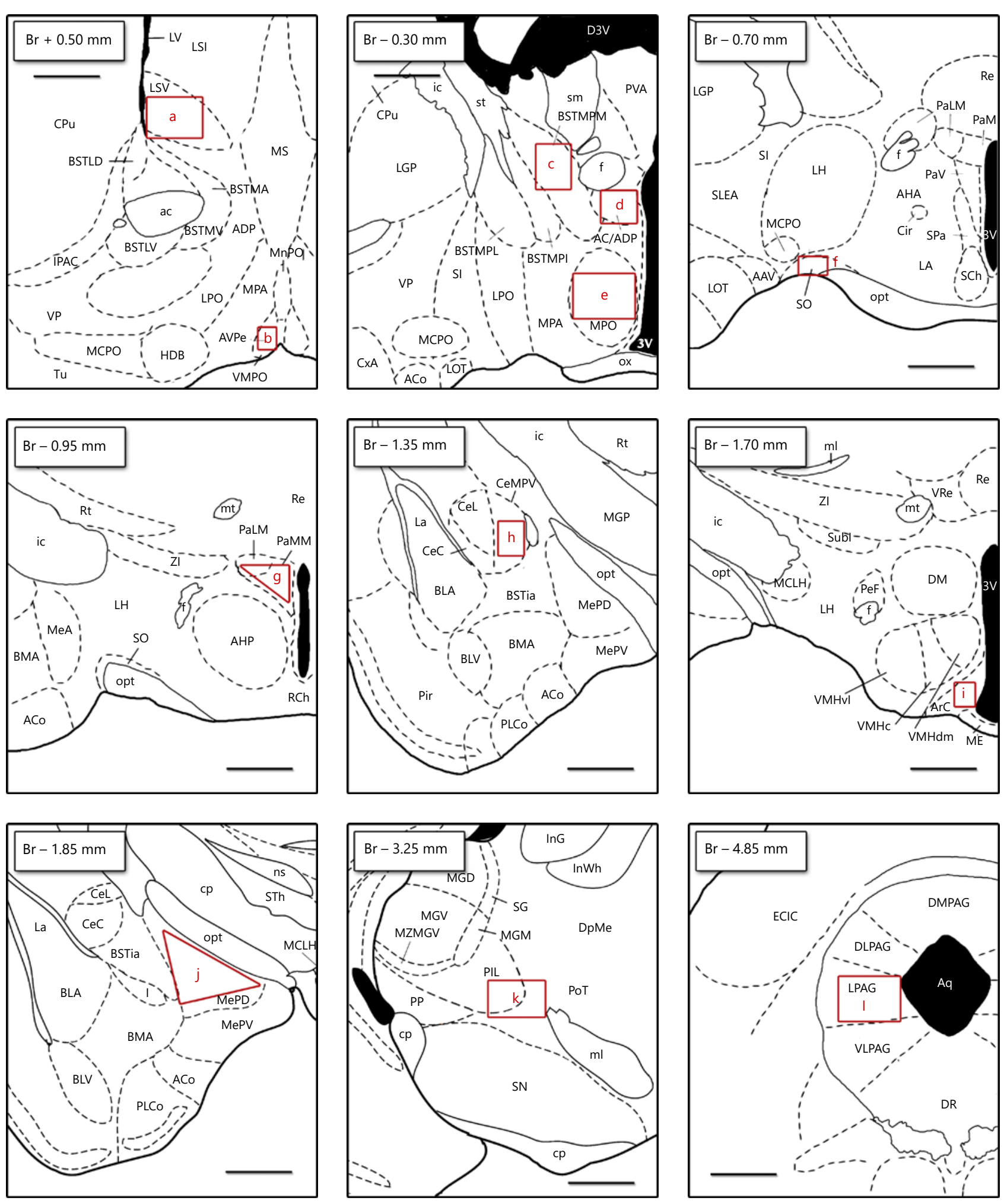

Fig. 2. Anatomical location of the sampling frames. Location of the frames in the nuclei where we sampled the density of pSTAT5-ir cells, with indication of the Bregma level, according to [33]. Red squares indicate the approximate size and location of the region analysed in each brain centre. pSTAT-ir, phosphorylated signal transducer and activator of transcription 5-immunoreactivity. 
Statistical Analyses

Quantitative data obtained in both experiments were statistically analysed using SPSS software package (IBM).

\section{Behaviour}

Concerning data on the MPRT, we first searched for possible inter-group differences (dams, STCs, LTCs, and virgins) in locomotion, anxiety, and interaction with pups, by statistically comparing the following variables: number of crossing across 3 parallel lines dividing the cage in 4 sectors, time in the centre of the cage, latency to first contact with pups, total time of pup contact, and number of barrier crossings. Since data for these variables were not normally distributed even after logarithmic transformation, we performed separate Kruskal-Wallis ANOVAs for each trial. This allowed us to discard differences between dams, comothers, and virgin females relative to pup approach/avoidance and motor abilities.

Then, we performed log rank (Kaplan-Meier) tests in search for significant differences in the distribution of the latencies to retrieve the first and the fourth pups of each of the 3 trials. This allowed comparing the performance of different females (dams, STCs, LTCs, and virgins) in the MPRT. Finally, we were interested in assessing the improvement of the experimental groups in the task (e.g., motor learning or increased motivation). Since latencies were not normally distributed among our experimental groups, we ran separate Wilcoxon signed-rank tests for matched pairs. To do so, we considered the latencies of each experimental animal to retrieve the first pup and compared the distributions of these latencies during the first trial with those in the second and third trials, respectively.

Differences in PRL Signalling in the Analysed Brain Centres

Differences in the density of pSTAT5-ir cells were statistically analysed independently for each one of the sampled nuclei. Whenever normality and homoscedasticity were fulfilled, we applied a one-way ANOVA. Otherwise, a Kruskal-Wallis non-parametric test was carried out. When these tests rendered significant differences, we further explored these differences using Dunnet's post hoc comparisons. We aimed at understanding the relative role of exposure to pregnancy hormones and to outer stimuli (mainly pup-derived ones), in PRL signalling revealed by pSTAT5-ir. Therefore, we used comothers as a reference group, to which dams and virgins are compared. The comparison of comothers with dams (both exposed to pup-derived stimuli) allows checking the effects of previous exposure to pregnancy hormones (occurring in dams, not in comothers). On the other hand, the comparison of comothers with pup-naïve virgins (neither of them exposed to pregnancy hormones), allows testing the role of pup-derived stimuli, to which comothers but not virgins are exposed.

\section{Correlation Analysis}

Besides the statistical analysis described above, we also explored the correlation between motivated maternal behaviour (cumulative latency to retrieve the first pups in the 3 trials; number of pups retrieved through the 3 sessions of MPRT) and the density of pSTAT5-ir cells. We did so globally for all the experimental females (dams plus comothers and virgins), as well as separately for each group, by means of Spearman correlation (behavioural data lacked normality), independently for each brain centre. This way we checked where in the brain PRL signalling correlates with maternal motivation in dams and comothers.

Neuroendocrinology of Maternal

Motivation
In addition, we also performed a cross-correlation analysis of the density of pSTAT5-ir cells between the different sampled nuclei in the brain of dams, STCs, and virgins. This allowed us to check whether pSTAT5-ir was coherently increased or decreased in the different brain centres in the different groups of females, as expected if it is reflecting circulating levels of PRL.

\section{Circulating PRL Levels in Comothers}

In the absence of variance homogeneity, serum PRL values of the samples of experiment 2 were statistically compared through a non-parametric Kruskal-Wallis ANOVA with multiple post hoc comparisons.

\section{Results}

\section{Experiment 1: Maternal Motivation and PRL}

Signalling

\section{Motivated Pup Retrieval Test}

First, we checked for possible differences in locomotion, barrier crossing, time in the centre of the cage, latency to approach pups, and the time that the experimental animals interacted with the pups behind the barriers. Locomotion and time in the centre of the cage showed no differences among groups (see online suppl. Fig. 1; see www.karger.com/doi/10.1159/000510038 for all online suppl. material). Regarding the interaction with pups, Kruskal-Wallis tests (data did not fulfil criteria for parametric ANOVA), revealed no significant differences during any of the 3 trials among experimental groups in the latency to cross the barrier $(p=0.126, p=0.518$, and $p=$ 0.101 for the 3 trials, respectively), the number of barrier crossings ( $p=0.247, p=0.843$, and $p=0.197)$, or the total time in contact with pups behind the barriers $(p=0.929$, $p=0.463$, and $p=0.137$ ) (Fig. 3). These results indicate that all 3 groups of females had equivalent access and interaction with pups, with no evidence of differential aversion or neophobia induced by the barriers (or the pups). They also indicate that dams, virgins, and comothers are equally able to climb the barriers back and forth.

Then, using the Kaplan Meier "survival" analysis, we explored differences in the distribution of the retrieval latencies for the first and the fourth pups in each trial between dams, comothers and virgins (Fig. 4). During the first trial, the survival distributions of the latency to retrieve the first pup (Fig. 4a) significantly differed between dams and STCs $\left(\chi^{2}[2]=4.194, p=0.041\right)$ as well as between dams and virgins $\left(\chi^{2}[2]=6.389, p=0.011\right)$ but not between dams and LTCs $\left(\chi^{2}[2]=1.556, p=0.212\right)$, comothers and virgins $\left(\chi^{2}[2]=1.0, p=0.317\right.$ for STCs; $\chi^{2}[2]=2.675, p=0.102$ for LTCs), or both kinds of comothers $\left(\chi^{2}[2]=0.678, p=0.410\right)$. Regarding retrieval of 
Fig. 3. Dams, comothers, and virgins show equivalent interactions with the barriers and pups in the MPRT. Bar histograms showing mean \pm SEM values (individual data are also plotted) of: the latency to establish the first contact with a pup (in seconds) (a); the number of barrier crossings into the compartment where pups were placed (b); and the total time of contact with pups behind the barriers (in seconds) (c). Kruskal-Wallis ANOVAs of these measures revealed no statistically significant differences among dams (red), STCs (blue), LTCs (striped blue), or pup-naïve virgins (green) in either of the 3 consecutive trials. This indicates equivalent access and interaction with pups, and absence of pup avoidance (e.g., pup-induced anxiety) in pup-naïve virgins. STCs, short-term comothers; LTCs, long-term comothers; MPRT, motivated pup retrieval test.
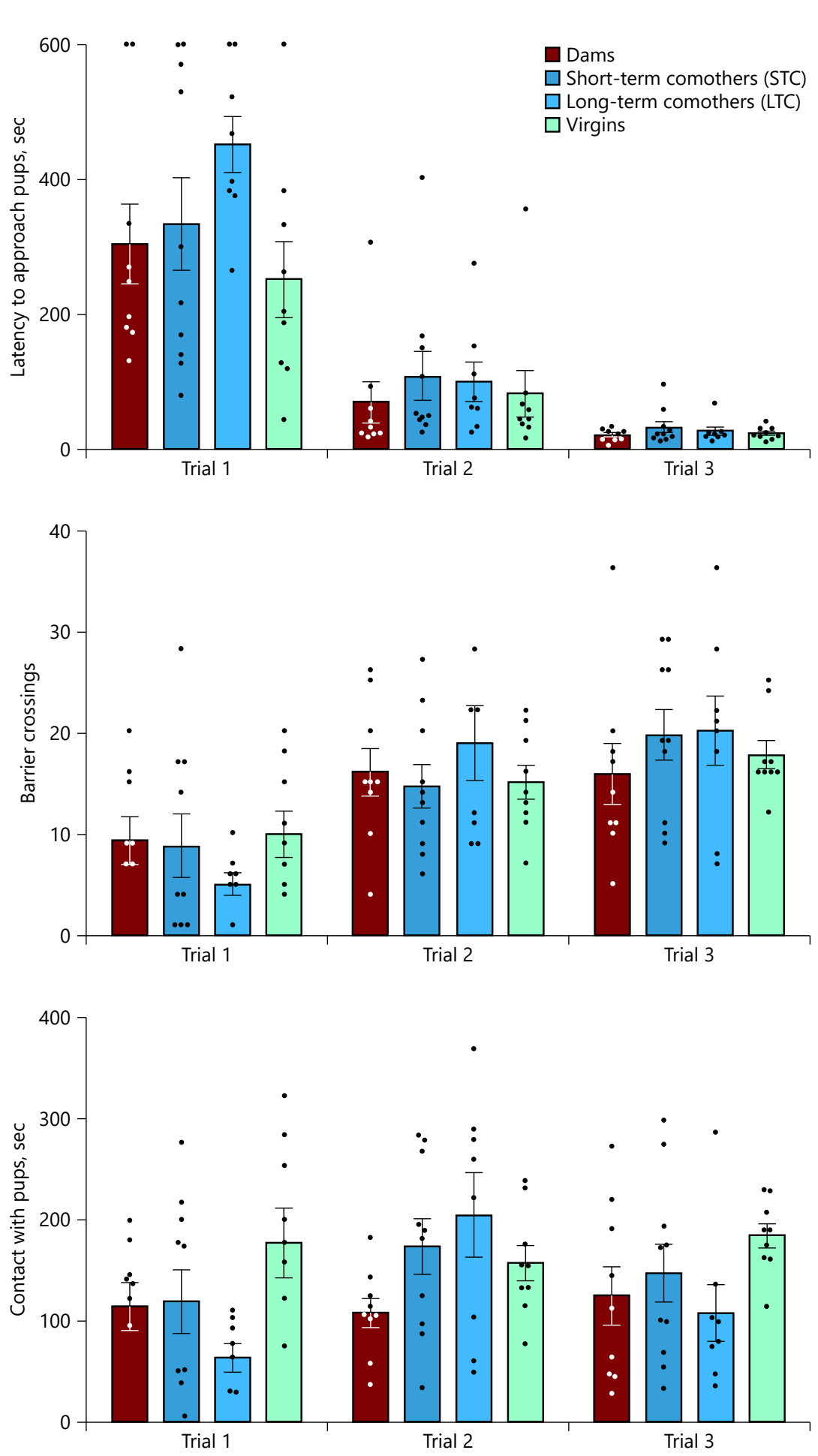


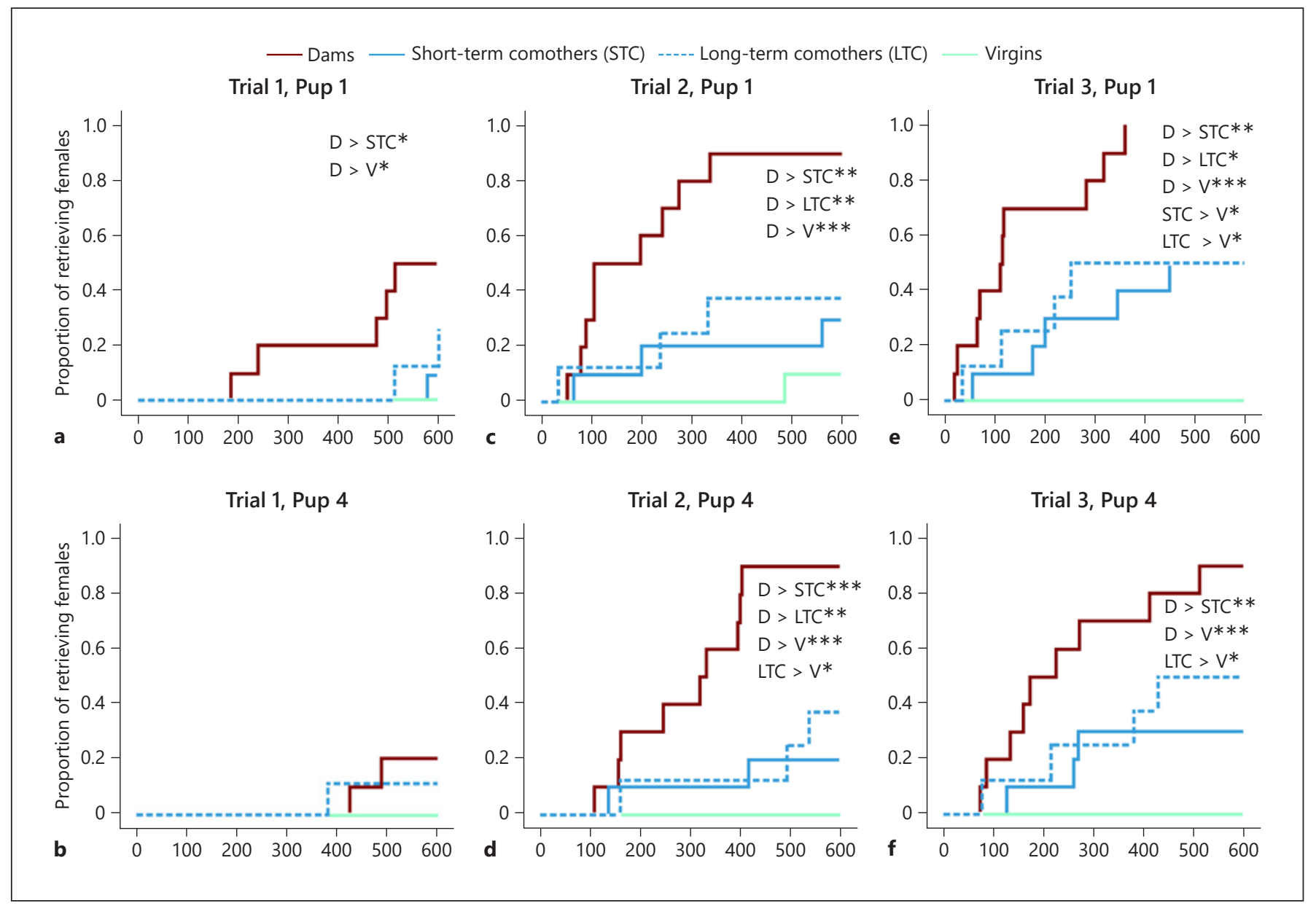

Fig. 4. Dams, comothers, and virgins perform differently in the MPRT. "Survival" plots indicating the cumulative proportion of dams (red lines), STCs (blue solid lines), LTCs (blue dotted lines), and virgins (green lines) successfully retrieving the first (upper row; a, e, f) and fourth pup (lower row; $\mathbf{b}, \mathbf{d}, \mathbf{f}$ ) through each of the three 10-min trials (columns; Trial 1: a, b; Trial 2: c, d; Trial 3: e,

the fourth pup (Fig. $4 \mathrm{~b}$ ), however, no significant differences between experimental groups were found $(p=0.146)$.

During the second trial, survival distributions of the latencies to retrieve the first (Fig. 4c) and fourth pup (Fig. 4 d) were both significantly different between dams and comothers (STCs: $\chi^{2}[2]=8.198, p=0.004$ for pup 1 and $\chi^{2}[2]=10.715, p=0.001$ for pup 4 ; LTCs $\chi^{2}[2]=$ $4.965, p=0.026$ for pup 1 and $\left.\chi^{2}[2]=7.553, p=0.006\right)$, as well as between dams and virgins $\left(\chi^{2}[2]=15.764, p<\right.$ 0.001 for pup $1 ; \chi^{2}[2]=16.989, p<0.001$ for pup 4$)$. Moreover, statistically significant differences appear between LTCs and virgins, only in latency to retrieve the fourth $\operatorname{pup}\left(\chi^{2}[2]=7.553, p=0.006\right)$ but no other significant differences were found between comothers and virgins $(p>$

Neuroendocrinology of Maternal Motivation f). Significant differences between experimental groups as revealed by log-rank (Kaplan-Meier) statistics are indicated for each trial: ${ }^{*} p<0.05 ;{ }^{* *} p<0.01{ }^{* * *} p<0.001$. MPRT, motivated pup retrieval test; STCs, short-term comothers; LTCs, long-term comothers; D, dams.

0.14 in every comparison), neither between both kinds of comothers $(p>0.499)$.

Finally, during the third trial, dams, comothers, and virgins differed significantly in many of the latencies to retrieve the first (Fig. 4e) and fourth pups (Fig. 4f). Dams showed statistically significant shorter retrieval latencies than virgins $\left(\chi^{2}[2]=21.837\left(p<0.001\right.\right.$ for pup $1 ; \chi^{2}[2]=$ $16.989, p<0.001$ for pup 4). Dams also differed from STCs (short-term, $\chi^{2}[2]=9.591, p=0.002$ for pup 1; $\chi^{2}[2]=6.981, p=0.008$ for pup 4$)$ but when compared to LTCs they differ in the retrieval of the first pup $\left(\chi^{2}[2]=\right.$ 4.577, $p=0.032$ ), whereas difference in the retrieval of pup 4 between dams and LTCs does not reach significance $\left(\chi^{2}[2]=3.422, p=0.064\right)$. Finally, when comparing 

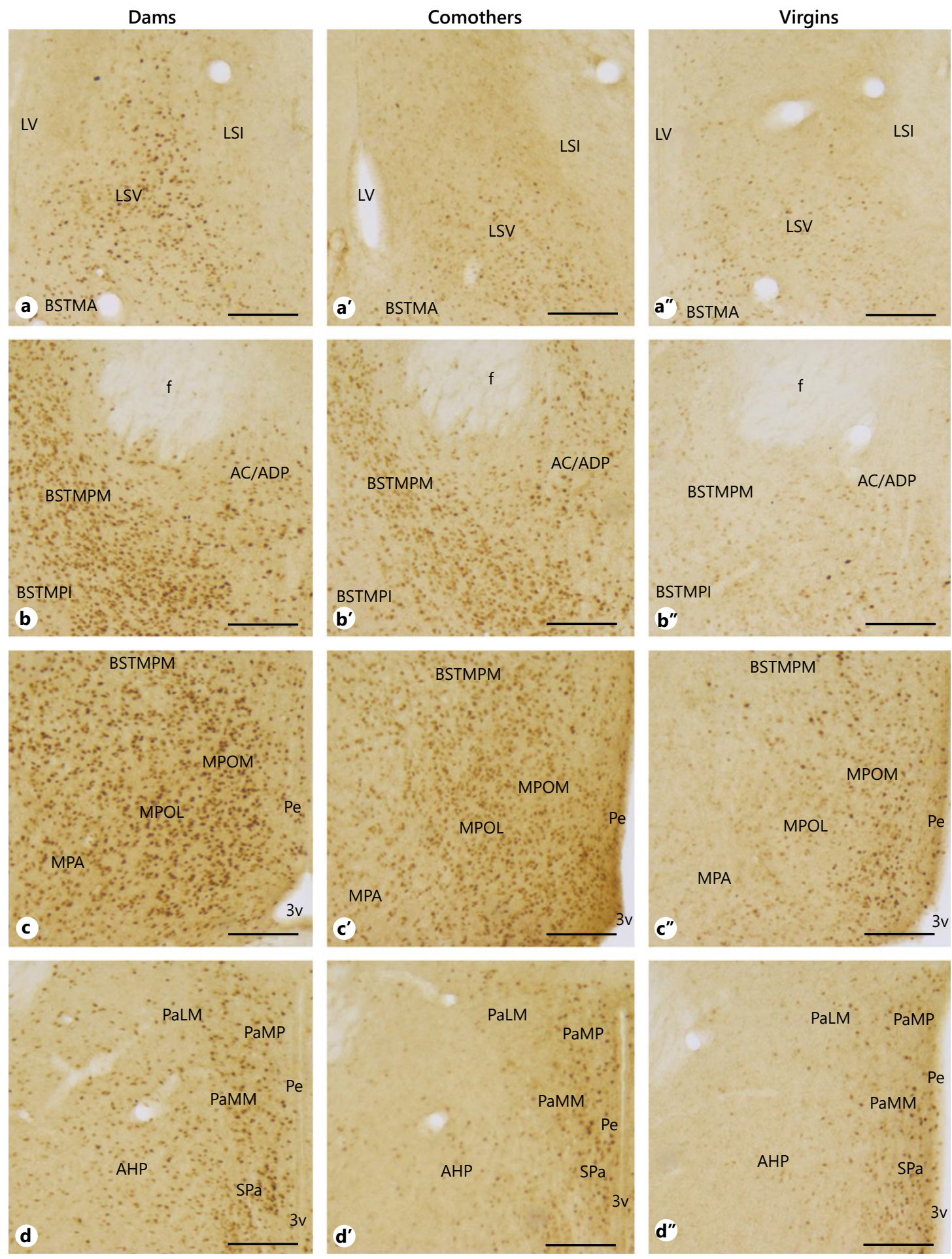

Fig. 5. Representative examples of pSTAT5 immunoreactivity in the brain of dams, comothers, and virgins. Photomicrographs showing pSTAT5 immunoreactivity in representative brain sections of dams (left) (short-term), comothers (centre), and pup-naïve virgin female mice (right). Sections correspond to the lateral septum (a-a", Breg$\mathrm{ma}+0.4 \mathrm{~mm})$, medial posterior BST and preoptic area (b-b", Bregma $-0.2 \mathrm{~mm}$ ), medial preoptic region (c-c"', Bregma $-0.2 \mathrm{~mm}$ ),
$\mathrm{Pa}$ (d-d', Bregma -0.85 mm), tuberal hypothalamus (e-e", Bregma $-1.80 \mathrm{~mm})$, medial posterior amygdala (f-f'", Bregma $-1.80 \mathrm{~mm}$ ), PIL (Bregma $-3.28 \mathrm{~mm}$ ) of the thalamus $\left(\mathbf{g}-\mathbf{g}^{\prime \prime}\right)$, and periaqueductal grey (h-h", Bregma $-4.80 \mathrm{~mm})$. Scale bars represent $150 \mu \mathrm{m}$. BST, bed nucleus of the stria terminalis; pSTAT5, phosphorylated signal transducer and activator of transcription 5; $\mathrm{Pa}$, paraventricular hypothalamic nucleus; PIL, posterior intralaminar thalamic nucleus.

(Figure continued on next page.) 

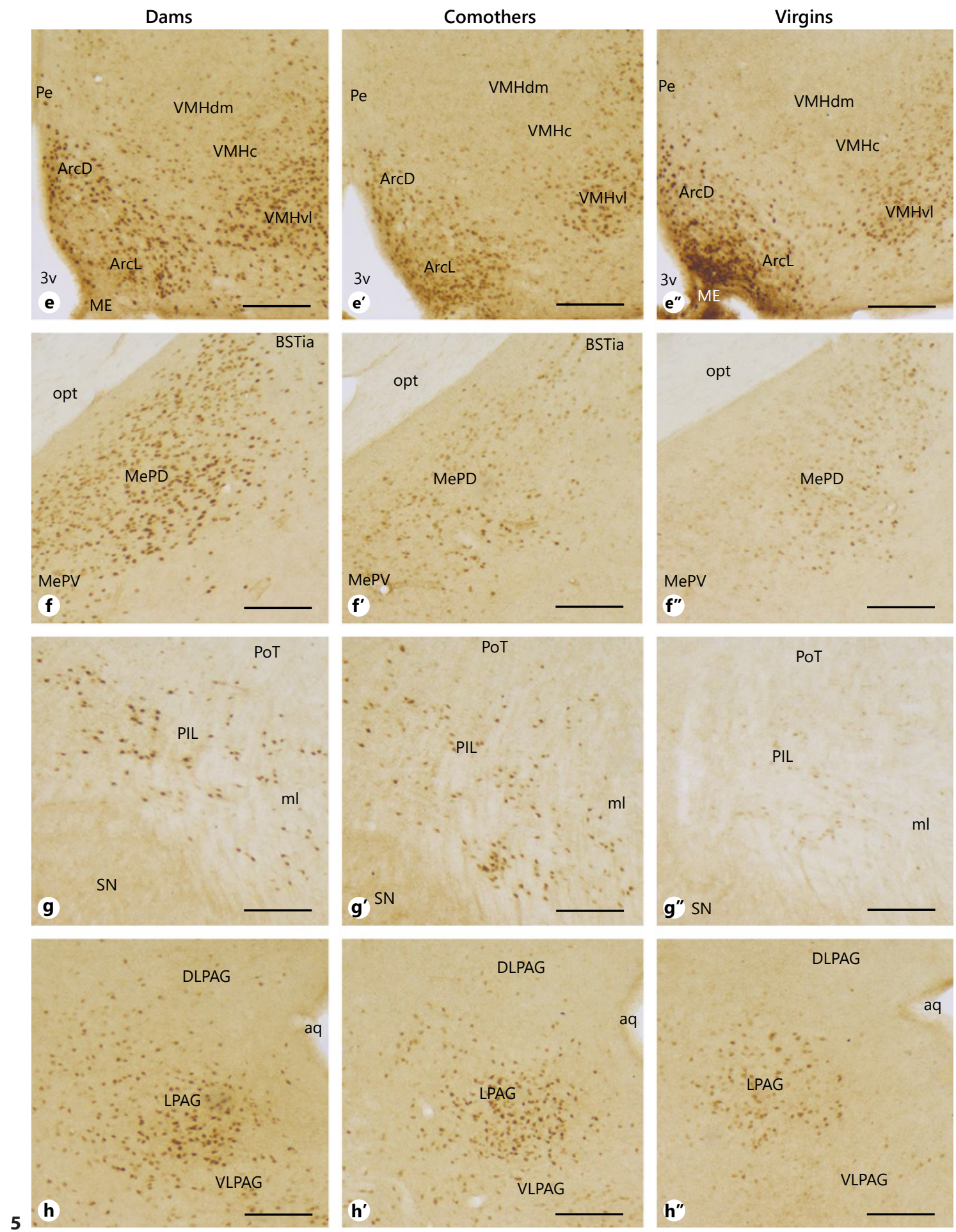


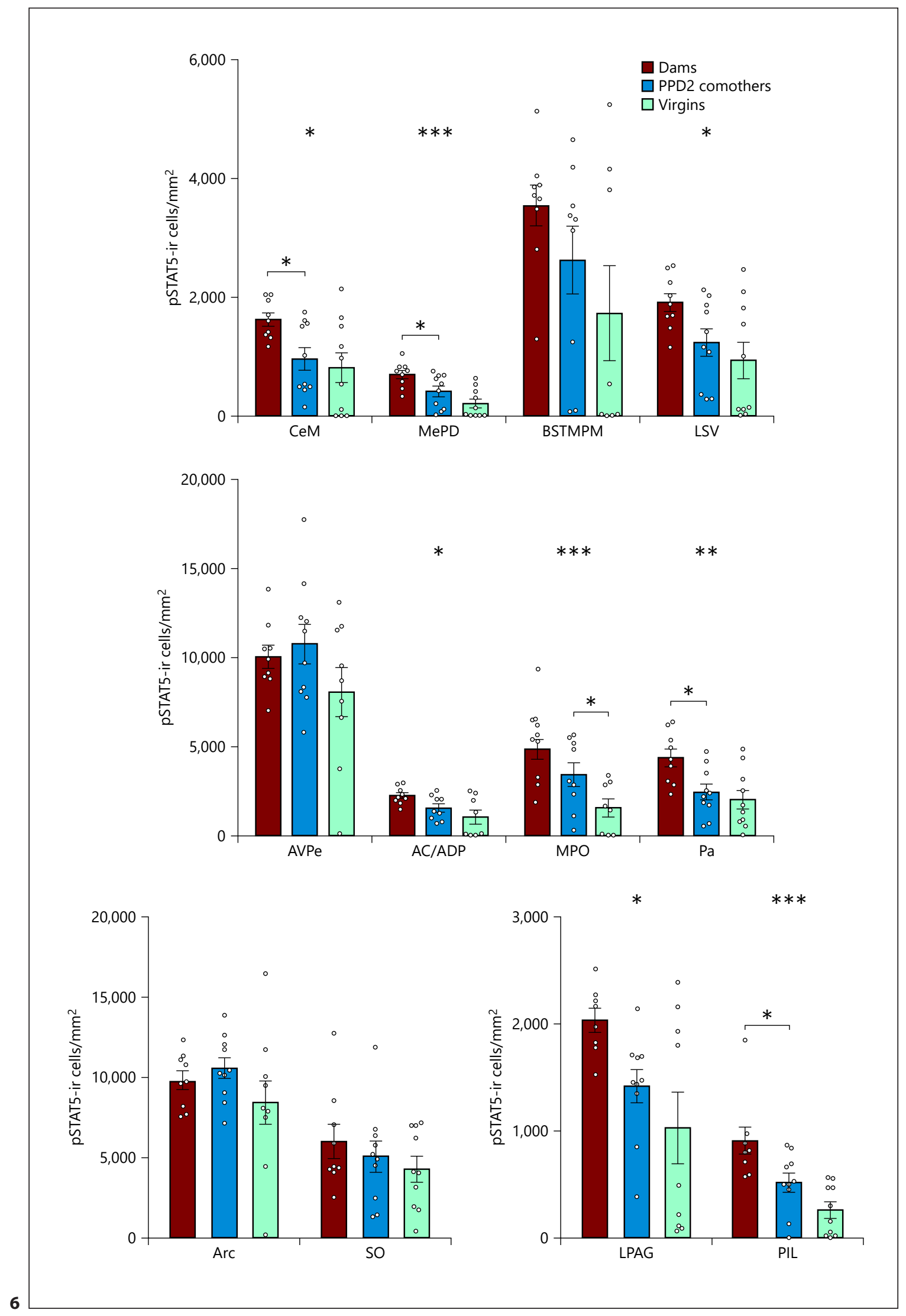

(For legend see next page.) 
virgins with comothers in this session, significant differences are found between virgins and LTCs $\left(\chi^{2}[2]=6.272\right.$, $p=0.012$, pup $1 ; \chi^{2}[2]=6.272 . p=0.012$, pup 4$)$, but differences with STCs are restricted to retrieval of the first $\operatorname{pup}\left(\chi^{2}[2]=6.389, p=0.011\right)\left(\right.$ for $\operatorname{pup} 4 ; \chi^{2}[2]=3.353$, $p=0.067)$. Thus, comparison of pup retrieval in the third session is suggestive of a certain higher degree of sensitization (e.g., maternal motivation) of long, as compared to STCs, although both groups of comothers showed similar latency to retrieve pups $(p>0.445)$.

The effects of maternal sensitization that occurred in comothers are illustrated by the results of the last trial, in which $50 \%$ (Fig. $4 \mathrm{e}$ ) of the comothers of both groups retrieved the first pup, and 30-45\% completed the whole task retrieving all 4 pups (Fig. 4f). By contrast, only a naïve virgin female retrieved a single pup in the whole trial.

Finally, we assessed the improvement of each experimental group in the motivated pup retrieval task by comparing first-pup retrieval latencies between trials 1 and 2 , and 1 and 3, by means of a Wilcoxon signed rank test for matched pairs. Since the previous analysis did not show any difference between short-term and longterm, their data were pooled for this analysis. When matching trials 1 and 2, only dams differed significantly in their latencies to retrieve the first pup $(Z=-2.666$, $p=0.008)$, whereas comothers $(Z=-1.604, p=0.109)$ and virgins $(Z=-1.000 p=0.317)$ did not. On the other hand, when comparing trials 1 and 3 , dams $(Z=-2.803$, $p=0.005)$ and comothers $(Z=-2.023, p=0.043) \mathrm{did}$ show statistically significant differences in their retriev-

Fig. 6. Quantitative analysis of pSTAT5 immunoreactivity in dams, comothers, and virgins. Bar histograms showing mean pSTAT5-ir density (cells $/ \mathrm{mm}^{2}$ ) \pm SEM in selected brain regions of dams (red), STCs (blue), and virgins (green). Individual data are also plotted. For each of the analysed nuclei, the location of the counting frames is indicated with red rectangles in Fig. 2. The results of the ANOVA (or non-parametric Kruskal-Wallis test; AC/ ADP, BSTMPM, CeM, LPAG, and LSV), are indicated with large asterisks above the histogram. Post hoc analysis was done using Dunnett's test comparing comothers with dams and with virgins (see text), and its results are indicated with small asterisks on the histogram. These analyses were applied independently to each brain region. ${ }^{*} p<0.05 ;{ }^{* *} p \leq 0.01 ;{ }^{* * *} p \leq 0.001$. STCs, short-term comothers; pSTAT5-ir, phosphorylated signal transducer and activator of transcription 5-immunoreactivity; AC/ADP, anterior commissure and anterodorsal preoptic nucleus; BSTMPM, bed nucleus of the stria terminalis posteromedial part; CeM, central amygdala medial division; LPAG, lateral periaqueductal grey; LSV, lateral septum ventral part; PPD, postpartum day.

Neuroendocrinology of Maternal Motivation al latencies, whereas virgins $\operatorname{did}$ not $(Z=0, p=1.0)$. These findings indicate that in a three-trial MPRT, dams rapidly improve in the task of retrieving pups (already during trial 2), comothers display a slower and weaker improvement (significant only in trial 3), and virgins show no improvement at all.

\section{Assessment of pSTAT5-ir in Dams, Comothers, and Virgins}

Like in previous studies [30, 31], in our material immunohistochemistry for pSTAT5 produced a defined, specific staining in the examined brain tissue (see Fig. 5), mostly restricted to the cell nucleus. To compare pSTAT5ir cell density in the selected brain regions in the 3 groups of females (dams, STCs, and pup-naïve virgins), we first performed a one-way ANOVA if the data fulfilled normality and homogeneous variance (Arc, AVPe, MePD, MPO, Pa, PIL, and SO), and a Kruskal-Wallis non-parametric ANOVA if not (AC/ADP, BSTMPM, CeM, LPAG, and LSV). When the ANOVA revealed significant differences between females, we compared comothers with dams and virgins using Dunnett's post hoc comparisons. The results of this analysis, illustrated in Figure 6, reveal significant intergroup differences in the density of pSTAT5-ir in telencephalic, amygdaloid, diencephalic, and midbrain nuclei, indicating generally higher PSTAT5ir densities in dams as compared to virgins.

Within the telencephalon, the amygdala showed significant differences in pSTAT5-ir density in both the $\operatorname{MePD}(F[2,26]=9.028 ; p=0.001)$ and CeM $(p=0.039)$ (Fig. 5f $-\mathrm{f}^{\prime \prime}$ ), but no differences in the medial extended amygdala (BSTMPM, $p=0.220$; Fig. 5b-b"). Post hoc analysis of this findings using Dunnett's test indicate that in both amygdaloid nuclei, comothers significantly differ from dams ( $p=0.038$ for the MePD; $p=0.047$ for the CeM) but not virgins $(p>0.1)$, thus indicating a crucial role of pregnancy hormones but not of outer social stimuli in PRL signalling. As for the septal region, the LSV (Fig. 5a- $\left.\mathrm{a}^{\prime \prime}\right)$ displayed significant differences $(p=0.048)$, with no difference of comothers with either dams or virgins $(p>0.1)$. This suggests that a combined effect of both stimuli (pregnancy hormones and pup stimuli) is shaping PRL signalling.

In the preoptic hypothalamus, significant differences were observed in the region comprised between the $\mathrm{AC} /$ ADP (Fig. 5b-b"; $F[2,29]=6.556 ; p=0.038$ ) and in the MPO (Fig. 5c-c"; $F[2,29]=9.026 ; p=0.001$ ), but not in the AVPe of the preoptic hypothalamus (AVPe; $F[2,29]=1.869 ; p=0.393)$. In the AC/ADP, comothers do not differ significantly from either dams or virgins. In

Neuroendocrinology 2021;111:805-830 817 
Table 1. Correlation between maternal behaviour (cumulative latency to retrieve the first pup and number of pups retrieved) and pSTAT5-ir in the different nuclei

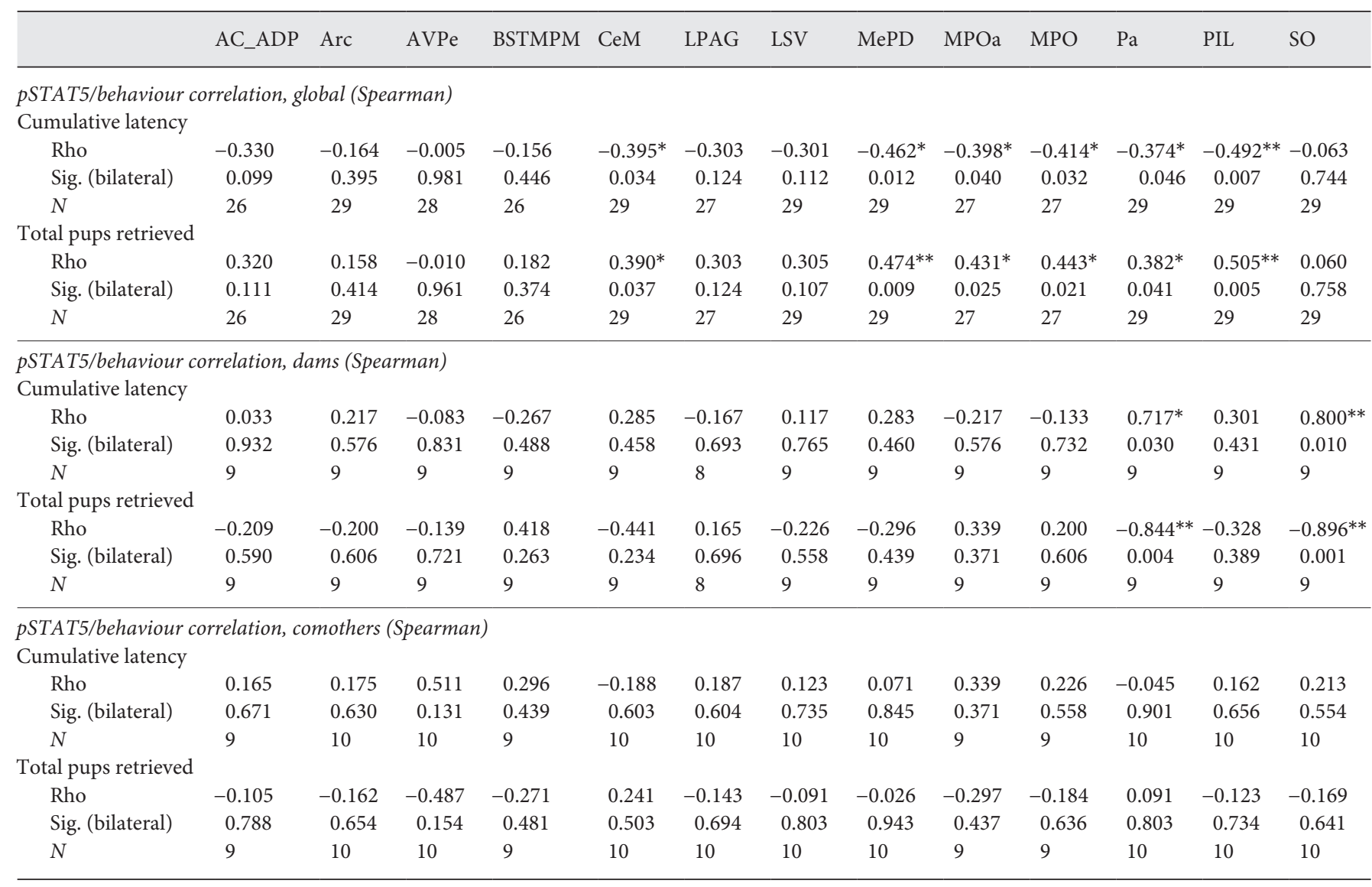

Brain-behaviour correlation. Analysis of correlation between performance in the MPRT (cumulative latency to retrieve pups in the 3 tests; total number of pups retrieved) and the density of pSTAT5-ir cells in the different nuclei analysed. Global correlation analysis (all animals included) is followed by individual analysis of the 3 groups of females (dams, comothers, and virgins). Grey indicates a significant correlation $(p<0.05)$. Spearman's rank correlation coefficients (rho) and their associated $p$ values are indicated in each cell of the table. ${ }^{*} p<0.05 ;{ }^{* *} p \leq 0.01$. pSTAT5, phosphorylated signal transducer and activator of transcription 5-immunoreactivity; AC/ADP, anterior commissure and anterodorsal preoptic nucleus; Arc, the arcuate nucleus; AVPe, anteroventral periventricular nucleus; CeM, central amygdala medial division; BSTMPM, bed nucleus of the stria terminalis posteromedial part; LPAG, lateral periaqueductal grey; LSV, lateral septum ventral part; MePD, medial amygdala posterodorsal division; MPO, medial preoptic nucleus; Pa, paraventricular hypothalamic nucleus; PIL, posterior intralaminar thalamic nucleus; SO, supraoptic nucleus; MPRT, motivated pup retrieval test.

contrast, in the MPO, comothers are similar to dams ( $p>$ $0.15)$ but significantly differ from virgins $(p=0.038)$. This suggests that outer stimuli, more than pregnancy hormones, are shaping PRL signalling.

In the anterior and tuberal hypothalamus, significant differences were found in the $\mathrm{Pa}(F[2,26]=6.365 ; p=$ $0.006)$, but not in the $\mathrm{SO}(F[2,26]=0.830, p=0.447)$, and $\operatorname{Arc}(F[2,26]=1.338 ; p=0.280)$. Dunnett's post hoc analysis of the Pa reveals that comothers differ significantly from dams ( $p=0.019)$ but not from pup-naïve virgins $(p>0.7)$, thus suggesting a crucial role of exposure to pregnancy hormones in shaping PRL signalling.
Finally, in the posterior thalamus and midbrain, the ANOVA revealed significant differences in the posterior intralaminar complex of PIL $(F[2,26]=10.979 ; p<0.001)$ and periaqueductal grey (LPAG; $p=0.019$ ). In the PIL, comothers differ significantly from dams but not from virgins, thus showing a crucial role of pregnancy hormones (but not pup- or dam's-derived outer stimuli) in shaping PRL signalling. In contrast, in the LPAG comothers do not differ significantly with either dams or virgins ( $p>0.1$ in both cases), thus suggesting again the need of a combined action of endocrine and outer stimulation in shaping of the response to PRL. 
Table 2. Correlation of PRL signalling between nuclei. Analysis of the correlation between the levels of pSTAT5-ir in the different nuclei analysed, using Pearson's correlation analysis

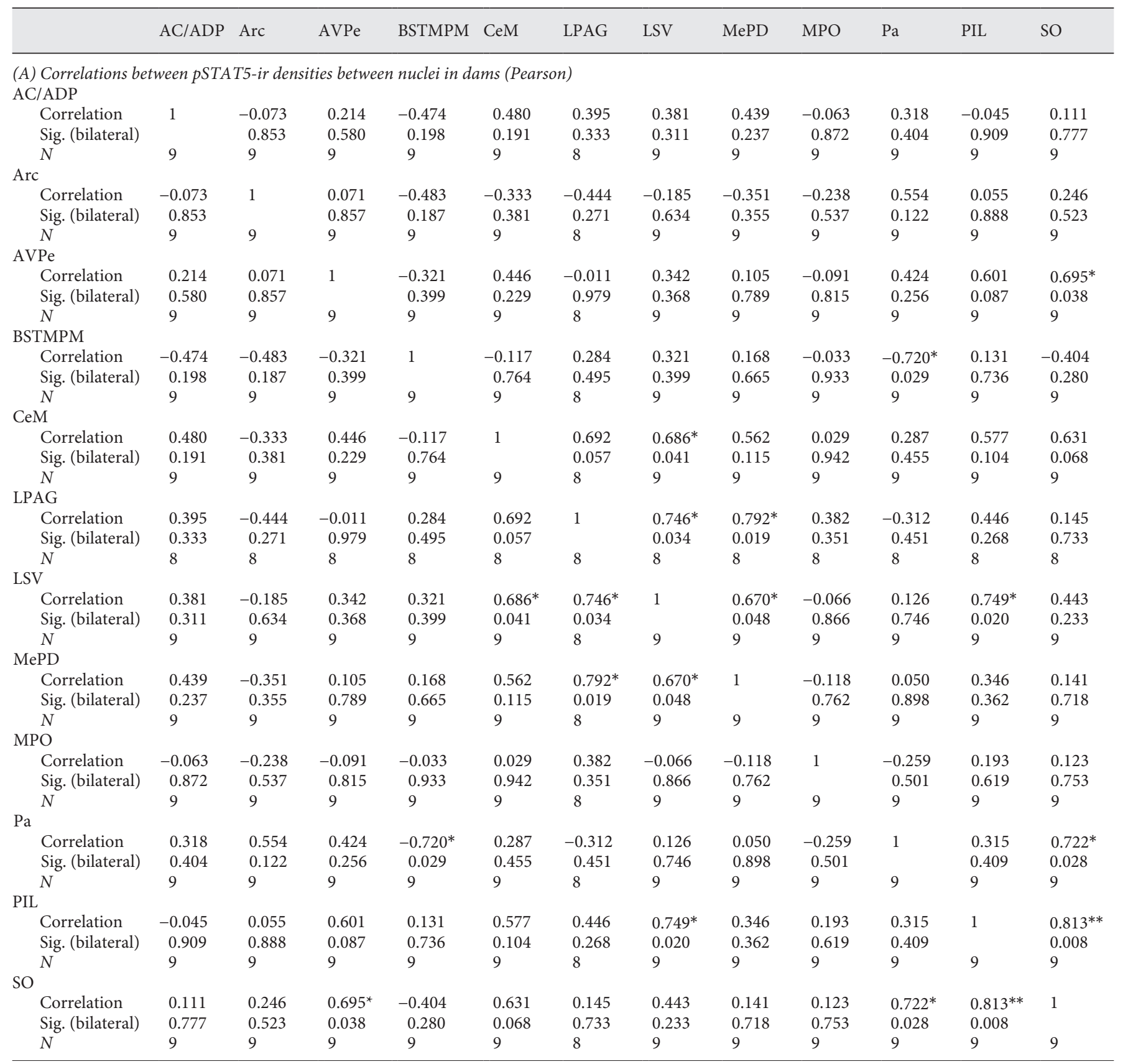

\section{Correlation Analysis: pSTAT5-Ir Cell Density and Behaviour}

Data on the density of pSTAT5-ir cells in each of the nuclei and maternal motivated behaviour (accumulated latency to pup retrieval in the MPRT; total pups retrieved through the 3 sessions of MPRT) belong to the same animals. To explore the relationship between maternal mo- tivation and PRL signalling we carried out an analysis of correlation between these variables using Spearman test (behavioural data did not follow a normal distribution). This was done first using data of all the animals for which both kinds of data were obtained, namely, dams, STCs, and pup-naïve virgins. 
Table 2 (continued)

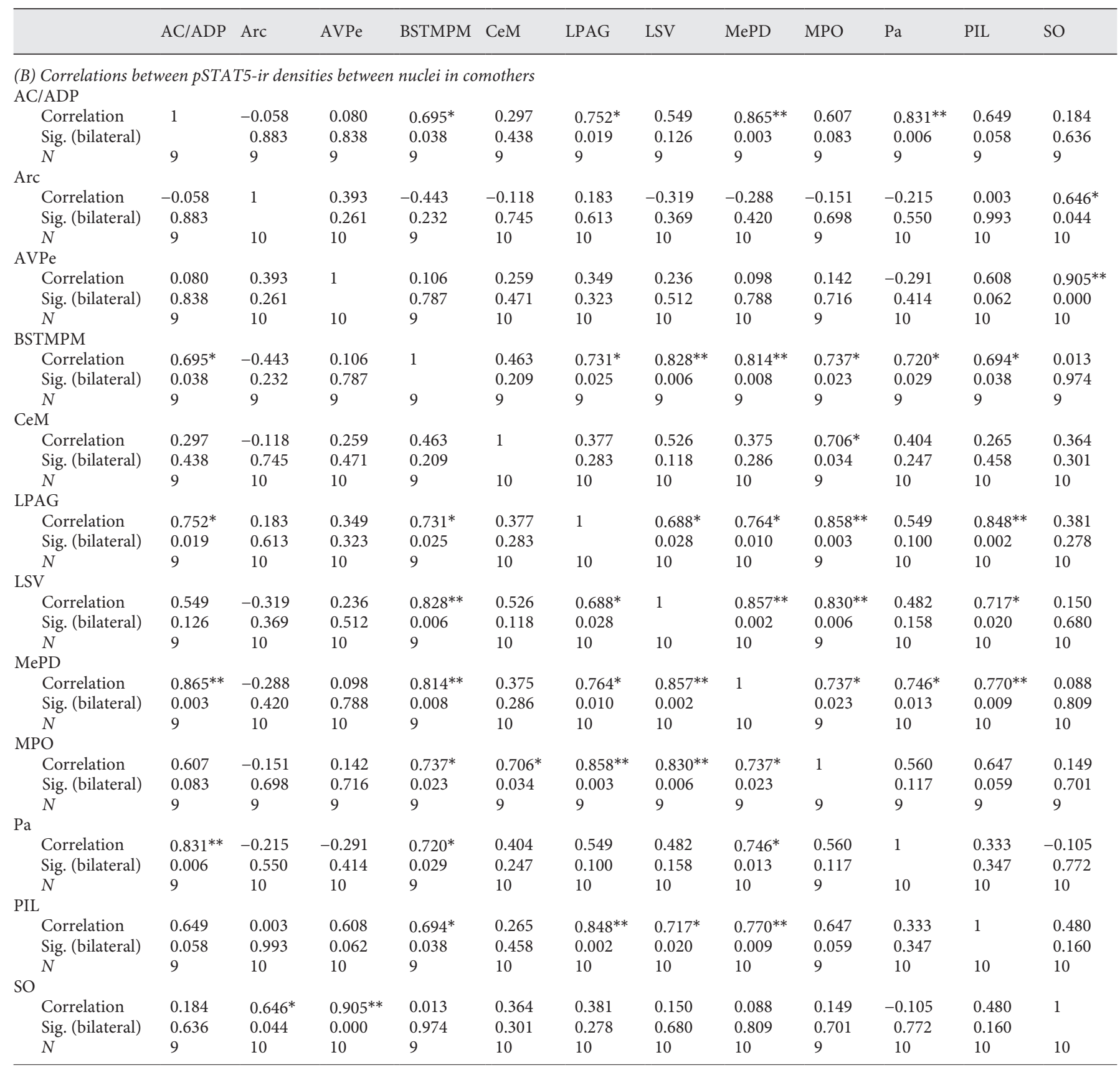

Table 1 shows the results of this correlation analysis. As expected, in those nuclei in which increased pSTAT-ir is observed in dams, pSTAT5-ir shows a mild, negative correlation with the cumulative latency to retrieve pups in all 3 tests, and a similarly mild positive correlation with the total number of pups retrieved throughout the 3 sessions of MPRT (CeM, MePD, MPO, Pa, and PIL). There are, however, 3 relevant exceptions: no correlation was observed in the LSV, LPAG, and AC/ADP. On the other hand, also as expected, in those nuclei showing no increase of pSTAT5-ir in dams, PRL signalling is not correlated with behaviour (SO, AVPe, Arc, and BSTMPM).

We also performed this correlation analysis within the groups of those females showing pup-retrieval in the MPRT, namely, in the group of dams and the group of comothers (separately). As Table 1 shows, in comothers 
Table 2 (continued)

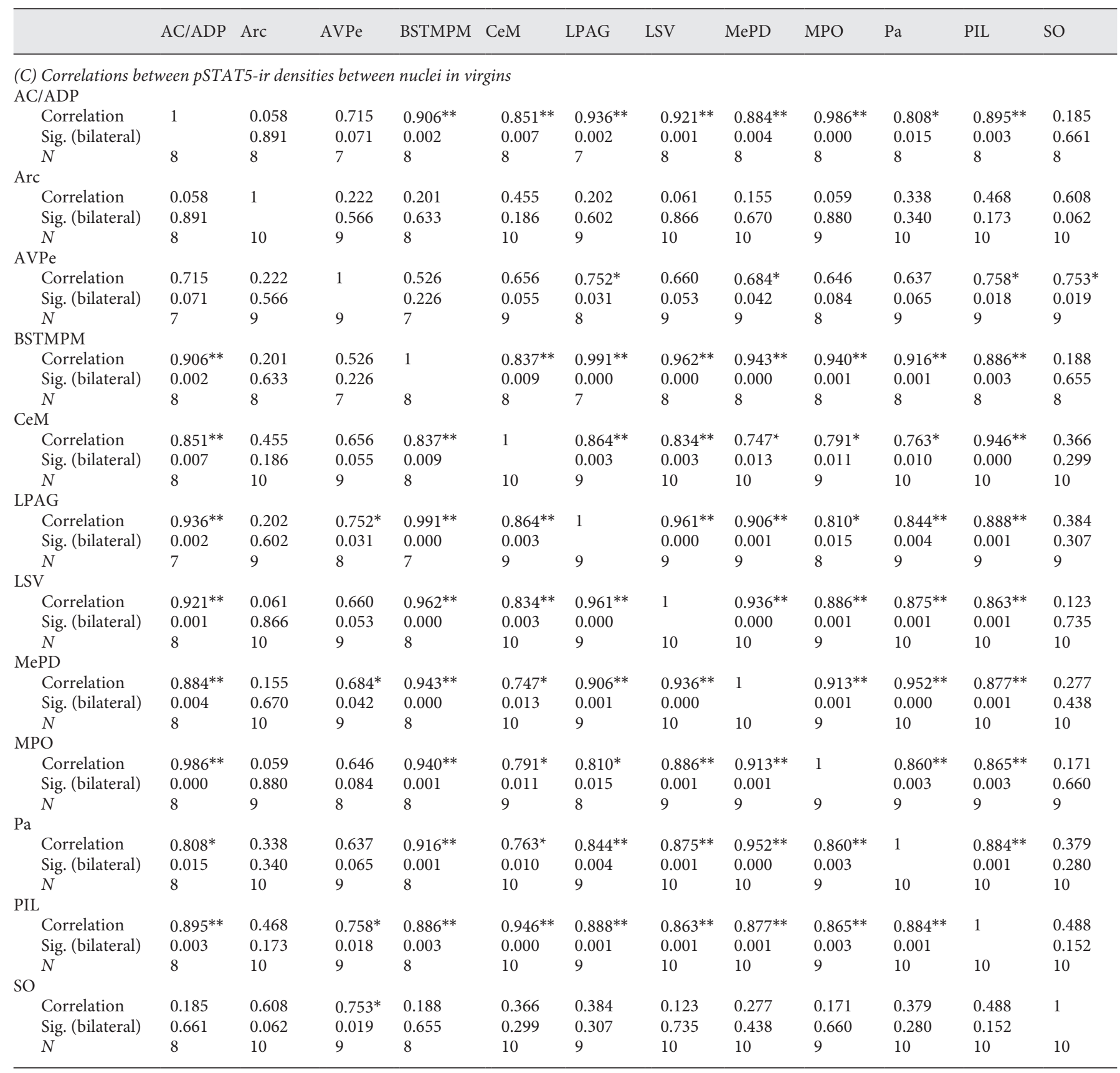

(A) shows the analysis for dams, (B) for comothers, and (C) for pup-naïve virgins, as indicated. In the upper right corner of each table, cells showing significant correlations $(p<0.05)$ are highlighted in grey. Spearman's rank correlation coefficients $($ rho $)$ and their associated $p$ values are indicated in each cell of the table. ${ }^{*} p<0.05 ; * * p \leq 0.01$. MePD, medial amygdala posterodorsal division; CeM, central amygdala medial division; BSTMPM, bed nucleus of the stria terminalis posteromedial part; AVPe, anteroventral periventricular nucleus; PRL, prolactin; pSTAT5, phosphorylated signal transducer and activator of transcription 5-immunoreactivity; MPO, medial preoptic nucleus; AC/ADP, anterior commissure and anterodorsal preoptic nucleus; Pa, paraventricular hypothalamic nucleus; SO, supraoptic nucleus; Arc, the arcuate nucleus; PIL, posterior intralaminar thalamic nucleus; LPAG, lateral periaqueductal grey. 


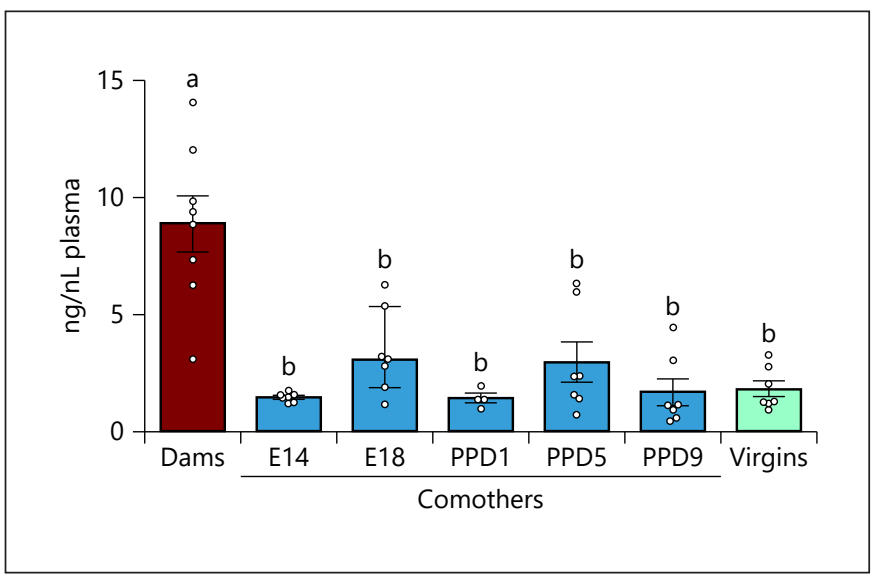

Fig. 7. Serum PRL levels in dams, comothers, and virgins. Bar diagram (mean \pm SEM) showing the values of serum PRL concentration in dams (red), comothers (blue), and virgins (green). Individual data are also plotted. The design included several groups of comothers subject to increasing periods of cohabitation with the accompanying maternal female and (in some groups) with her pups. Accompanying pregnant females were cohoused with comothers in pregnancy day 10 (E10) and blood samples were taken from comothers (from separate groups) at E14, E18, PPD1, PPD5, and PPD9, respectively. A group of pup-naïve virgins is included as control, and a group of dams as a positive control (PPD5). All serum PRL measurements were conducted with a commercially available PRL ELISA kit (see Material and Methods section). Nonparametric Kruskal-Wallis ANOVA revealed highly significant differences between females $(p<0.001)$ and post hoc comparisons revealed significantly higher serum PRL levels in lactating dams as compared to pup-naïve virgins or comothers. On the other hand, no significant differences appear between virgins and comothers. Differential letters indicate a significant difference among groups. PPD, postpartum day; PRL, prolactin.

no behaviour-pSTAT5 correlations appear in any of the analysed nuclei. By contrast, in dams, pSTAT5-ir cell density is strongly and significantly correlated with behaviour in the $\mathrm{Pa}$ and $\mathrm{SO}$, and no correlation is observed in any of the remaining nuclei. Surprisingly, in the $\mathrm{Pa}$ and SO of dams, pSTAT5-ir cell density is positively correlated with latency and negatively with the number of retrieved pups. In other words, the higher the density of pSTAT5-ir cells in the $\mathrm{Pa}$ and $\mathrm{SO}$, the slower is pup retrieval (higher latency, lower number of pups retrieved) in the MPRT.

\section{Correlation Analysis: Inter-nuclear Cross Correlation in pSTAT5-Ir Cell Density}

Finally, we performed a correlation analysis between levels of pSTAT5-ir observed in the different nuclei analysed, independently in all 3 groups of females. Table 2 summarizes the results obtained. The main output of this analysis is that in virgin females, most nuclei show positive, significant cross-correlation. The main exceptions are the Arc and the SO, which only show correlation with the AVPe. On the other hand, the AVPe shows correlations with the remaining nuclei, although in many instances there is a simple trend that does not reach significance. For the remaining nuclei (LSV, MePD, BSTMPM, AC/ADP, MPO, PIL, and LPAG) pSTAT5-ir displays highly significant positive crosscorrelation.

These results in pup-naïve virgins contrast with those observed in comothers and, especially, in dams. The number of correlations observed is much lower in those females. In Table 2, significant correlations are highlighted in grey $(p<0.05)$, and it can be observed that whereas for pup-naïve virgins (upper table), 40 out of 66 cells of the table show significant positive correlations, in comothers (middle), and dams (lower table) the number of correlations is reduced to 23 and 9 , respectively.

\section{Experiment 2: Serum PRL in Dams, Comothers, and Virgins}

After logarithmic transformation, data on serum PRL levels in dams, comothers (E14, E18, PP1, PP6, and PP9) and virgins (see Fig. 7) achieved normality but not homoscedasticity. Therefore, a nonparametric KruskalWallis analysis revealed significant differences in serum PRL levels between females $\left(\chi^{2}[6]=42.147, p<0.001\right)$. Post hoc comparisons showed that dams had significantly higher levels of serum PRL than comothers and virgins ( $p$ values between 0.042 and 0.000 ), whereas comothers and virgins did not differ significantly from each other $(p \geq 0.259)$.

\section{Discussion}

In the present work, we have analysed the importance of motivation in maternal behaviours in mice. For this purpose, we have designed a novel variant of the pup retrieval test that circumvents the limitations of the standard pup retrieval paradigm to evaluate motivational components of maternal behaviour, the MPRT. We report a high motivation to retrieve pups in dams, as well as increased motivation for pups in sensitized virgin females. In addition, we have explored the role of PRL in the induction of maternal motivation in dams and sensitized virgin females, and the brain centres where this PRL action might occur. 
In this section, we will first discuss the validity of the new pup retrieval paradigm employed in this work and comment on the findings resulting of this test. Then, we evaluate the putative role and interaction of pup-derived stimuli and endocrine signals (PRL) in the onset of proactive maternal responses, in the context of currently accepted models for the functional neuroanatomy of maternal behaviour.

\section{MPRT: A Novel Paradigm to Assess Maternal Motivation}

Assessment of maternal motivation requires the inclusion of activational aspects of motivation [37]. A limitation of conventional pup retrieval tests performed in the home cage in this sense is that they do not allow discriminating differences in motivation between dams and virgin females, as this task demands little effort and only moderate levels of motivation. This would explain the common occurrence of pup retrieval in unexperienced virgin females using the regular pup retrieval test (about $25 \%$ [16]) and its increase with further experience, reaching levels similar to those of dams after exposure to pups for a few hours $[15,16]$, or as a consequence of cohabitation with a dam and its litter (comothers [14]).

Previous works assessing maternal motivation [29] used alternative strategies in which access to pups posed a challenge, for instance, facing a novel T-maze where the pups are placed [15, 38-41], in which females have to overcome the anxiety associated to a new environment. Since anxiety is reduced in dams as compared to virgin females [42], the T maze paradigm might not allow properly discriminating anxiety-related and motivational components of maternal behaviour (but see Stolzenberg and Rissman [15], who discarded differences in anxiety between lactating dams and pup-experienced virgins).

To circumvent these limitations, we have developed the MPRT, which confronts the tested animals with an additional effort-demanding task (climbing a barrier back and forth). The female has to choose between staying with the 4 pups that remain in the nest (low effort, low reward) or retrieving the displaced 4 pups to the nest (very high effort) to become in contact with the 8 pups in the nest (high reward). The low effort/low reward versus high effort/high reward two-choice test has been used before in rodents to assess motivational aspects of behaviour and their dependence on dopaminergic neurotransmission in the nucleus accumbens [18]. Drugs reducing motivation, such as tetrabenazine (an inhibitor of the vesicular monoamine transporter, VMAT-2), disbalance this test in favour of the low effort/low reward option
[43]. Therefore, our results indicate that the motivation of dams for pups is very high, whereas maternal sensitization occurring in comothers involves an increase of maternal motivation that, however, does not reach the levels of dams.

In our paradigm, females do not leave their home cage to retrieve pups, minimizing anxiety. First, data presented in Figure 3 support that the barriers introduced in the home cage of the females during the MPRT do not induce significant neophobia. In addition, when reintroduced in their home cage for the first session of the MPRT, we have not observed differences in behavioural measures commonly used to measure anxiety (online suppl. Fig. 1) [44]. Thus, our data suggest that anxiety is not a relevant factor in the MPRT; pups are not apparently aversive for those animals having no previous experience with pups (pupnaïve virgins) or with these particular pups (LTCs that received "foster" pups); and all the experimental groups displayed equivalent motor performance or physical strength, thus discarding another potential bias for our results. Therefore, differences in retrieval performance found among experimental females can be attributed to motivation. Further, improvement of performance in the MPRT through the 3 sessions is also a proof of the reinforcing properties of pups to dams and, to a lesser extent, comothers.

In summary, our data validate the MPRT as a suitable paradigm to study maternal motivation. The fastest and largest improvement displayed by dams, as compared to comothers, demonstrates that pups are more reinforcing to dams than to comothers, whereas comparatively, pups do not constitute a strongly rewarding stimulus for pupnaïve virgin females.

Maternal Motivation in Dams, Comothers, and Virgin Females: Role of Hormones and Outer Stimuli

The MPRT is a difficult task for an adult female. The first test day, only $50 \%$ of the dams retrieve the first pup and just a $20 \%$ of them are able to retrieve all 4 pups during the 10-min session. Even so, they significantly differ from virgin females and STCs already in this session (Fig. 4), evidencing that endocrine inputs of pregnancy and lactation are essential for the development of full maternal motivation [10]. This is reinforced by the quicker and more complete learning displayed by dams through the second and third sessions. Concerning comothers, despite initially displaying no or delayed pup retrieval (Fig. 4), they improved progressively in subsequent trials, and by trial 3, they displayed consistent retrieval performance, differing significantly from pup-naïve virgins 
which underwent short (10 $\mathrm{min})$ exposure periods to pups only during the tests (Fig. 4). This finding indicates that prolonged exposure to a dam and her pups is also able to induce certain levels of motivation for pups, which contributed to learning the task, although not as effectively as its synergistic action with endocrine signals of pregnancy and lactation (dams). Therefore, in contrast to what the use of regular pup-retrieval tests might suggest [13], virgin female mice are not spontaneously fully maternal but require a pup-induced maternal sensitization to exhibit full maternal behaviour including enhanced motivation for pups $[15,16]$.

Our model of maternal sensitization, the comother, entails exposure of a virgin female to stimuli derived from pups and their mother (a pregnant, parturient, and lactating female), so that stimuli of the accompanying mother might be relevant for the observed process of maternal sensitization of comothers. This sensitization could provoke endocrine changes, such as increases in PRL, similar to those occurring in fathers in biparental species $[34,45]$. However, the results of our experiment 2 indicate that comothers show no increased levels of circulating PRL during either pregnancy or postpartum period of their mate. Nonetheless, we assume that even if stimuli emitted by the accompanying dam might be also relevant for maternal sensitization of comothers, interaction of comothers with pups during postpartum has a pivotal role in sensitization process, as demonstrated previously $[15,16]$.

We also evaluate the effect of the length of exposure to pups in the sensitization of comothers, by comparing 2 lengths of exposure to pups prior to the beginning of the MPRT (2 and 4 days, in STCs and LTCs, respectively). There are subtle differences between STCs and LTCs that may suggest a somewhat increased motivation in the latter. Thus, analysis of the retrieval of the fourth pups during the third session of the MPRT reveals that differences between dams and LTCs do not reach significance $(p=0.064)$, whereas STCs and pup-naïve virgins do not differ significantly either $(p=0.067)$. Similarly, LTCs do not differ significantly from dams in the first session of the MPRT. However, when directly comparing LTCs with STCs, there are no significant differences in pup retrieval between both groups. This suggests a certain ceiling effect regarding the effects of stimuli of pups and/or the dam, with 2 days of postpartum prior to the onset of the MPRT being sufficient to significantly facilitate the increase in motivation for pups. It also reveals that exposure to pups, even a long one, is not able to induce the high maternal motivation observed in dams, which clearly requires the action of hormones during pregnancy, parturition, and/or lactation.

\section{Motivated Maternal Behaviour and PRL Signalling}

In accordance with previous studies [30], our data indicate that most of the nuclei of the so-called sociosexual brain network, the circuitry encoding the expression of social behaviours [46], display increased pSTAT5-ir in dams, including the LSV, MePD, MPO, AC/ADP, CeM, $\mathrm{Pa}$, and LPAG. As indicated in previous works, moreover, these data are consistent with the distribution of PRL receptors in the brain of mice $[31,47]$. Our data differ, however, from those of our previous study in showing no differences between dams and virgins in the levels of pSTAT5-ir in the SO, a conflicting result for which we have no clear explanation. In addition, in this work, we have observed significantly increased pSTAT5-ir levels in the PIL of dams, which was not observed in our previous analysis [30], a difference that we attribute to the larger number of animals employed in this work. Even so, neither the AVPe nor the Arc displays differences in PRL signalling between dams and virgin females. The Arc and AVPe include the tuberoinfundibular dopaminergic and periventricular hypothalamic dopaminergic participating in the negative feedback control of the hypophyseal PRL $[48,49]$. In this context, dams (showing higher levels of circulating PRL, see Fig. 7) would be expected to have increased pSTAT5-ir. However, hyperprolactinaemia during lactation is due, at least in part, to reduced PRL negative feedback derived from a reduction of STAT5PRL signalling in tuberoinfundibular dopaminergic neurons [50] mediated by upregulation of the expression of CIS (cytokine-inducible $\mathrm{SH} 2$ domain-containing protein). This might explain why, in spite of their hyperprolactinemia (see Fig. 7), dams show no higher levels of pSTAT5-ir than virgin females in the Arc and AVPe.

The analysis of correlation between motivated maternal behaviour (number of pups retrieved and cumulative latency to retrieve the first pup) and the density of pSTAT5-ir in the nuclei under scrutiny allows mapping the brain centres where PRL action is critical for modulating motivational aspects of maternal behaviour. Our results reveal that only a fraction of the nuclei where pSTAT5-ir density is increased during postpartum [30, 32] shows correlation between PRL signalling and behaviour. This occurs in the CeM, MePD, MPO, Pa, and PIL. Even though different factors may influence PRL signalling in the female brain, our data specifically indicate that the more the PRL signalling occurs in these nuclei, the higher the motivation of the females for pups. Although correlation does not demonstrate causal relationships, evidence in rats and mice indicates a causal role of PRL in the induction of proactive maternal behaviours $[21,51$, 
52]. This evidence, together with our findings, strongly suggests that action of PRL in these nuclei is part of the mechanisms underlying the increased motivation of dams for pups. By contrast, in the LSV and LPAG, postpartum females display increased pSTAT-ir [30, 32], but there is no correlation between PRL signalling and motivation to retrieve pups. This suggests that in these brain centres, PRL action is likely involved in other aspects of maternal behaviour and/or physiology not specifically related to motivation for pups (e.g., oxytocinergic neurotransmission in the LSV mediates the reduction of social anxiety occurring during motherhood in mice [53]).

Our findings fit previous studies in rats indicating that PRL or placental lactogens infused intracerebroventricularly or locally in the preoptic area induce maternal care in steroid-primed virgin rats [21]. In addition, our findings also suggest that action of PRL in nuclei outside the preoptic area might be relevant for the expression of maternal motivation. In lactating rats, PIL cells show increased expression of a tuberoinfundibular peptide of 39 residues [54], and these tuberoinfundibular peptide of 39 residue-expressing cells project to (among other targets) the preoptic region, where they activate parathyroid hormone 2 receptor, inducing maternal motivation for pups [55] as revealed by pup-induced place preference. The MePD is likely to be part of this circuitry, as it projects massively to the MPO [56] and receives ascending projections from PIL [57].

The functional model by Numan and Woodside, based on the evidence in the rat ([24], but see also $[58,59])$, proposed a specific neuroanatomical substrate of the influence of the medial preoptic area on maternal motivation. According to it, pup stimuli would activate the preoptic pathways to the ventral tegmental area, thus stimulating mesocorticolimbic dopaminergic pathway in the maternal female, triggering proactive maternal behaviours. In rats, this only happens if the preoptic area undergoes hormonal priming during pregnancy, which is induced by lactogenic hormones and other endocrine signals of motherhood [24, 60, 61].

Recent data in mice have depicted a similar role of the medial preoptic area in motivation for pups. Using optogenetics, activation or inhibition of specific galaninergic preoptic cells projecting to the ventral tegmental area [29] has been shown to increase or reduce (respectively) motivation to be in touch with pups, using a behavioural paradigm similar to ours (a climbable barrier separating the female from the pups). There is solid evidence indicating that the medial preoptic area and some of its main inputs, such as the PIL and MePD [56], undergo a hormone-me-

Neuroendocrinology of Maternal Motivation diated priming process in the female mouse, involving the action of placental lactogens during prepartum [30] and of PRL during postpartum [30, 32, 62]. The circuit composed by the MPO, PIL, and MePD shows a clear positive correlation between pSTAT5-ir cell density and motivation for pups, thus strongly suggesting that PRL signalling at this level is critical for development and/or maintenance of maternal motivation.

The remaining 2 nuclei where pSTAT5-ir is correlated with performance in the MPRT, $\mathrm{Pa}$, and CeM, probably participate also in aspects of maternal behaviour relevant for pup retrieval. Knobloch and her collaborators [63] demonstrated in the rat that $\mathrm{Pa}$ oxytocinergic cells projecting to CeM are involved in the downregulation of fear/ anxiety characteristic of motherhood. Our data suggest a role of PRL in this effect of oxytocin in the Pa-CeM pathway, although its impact on performance on the MPRT is paradoxical. Thus, when the correlation between pSTAT5ir and MPRT scores is analysed only in the group of dams, there is a highly significant negative correlation between pSTAT5-ir cell density in the $\mathrm{Pa}$ (and SO) and maternal motivation in the MPRT (see Table 1): the more PRL signalling in these nuclei, the higher the latency to retrieve pups in the MPRT and, consequently, the lower the total number of pups retrieved through the 3 test sessions. This fits the findings by Neumann and her group ([64]; reviewed by [65]) on the maternal behaviour displayed by dams of high- (HAB) versus low-anxiety (LAB) selected lines of rats. High-anxiety rat dams retrieve pups earlier and are able to retrieve more pups in a given test time than low-anxiety ones, and similar results were reported by the same group in CD1 mice [66]. This can be interpreted as anxious females displaying a more protective maternal style, as observed in primates [67]. Our data thus suggest that during motherhood, the action of PRL onto Pa (and $\mathrm{SO}$ ) cells would promote a decrease in anxiety (an effect maybe mediated by oxytocin acting onto the CeM) and, consequently, the exhibition of a less protective maternal style, with longer latency to retrieve pups.

Our correlation analysis has allowed mapping a series of nuclei where PRL signalling is relevant for the motivation to retrieve pups to the nest. Experimental studies altering PRL signalling locally in these nuclei are needed to clarify the role of PRL in tuning the brain for motherhood.

\section{Maternal Sensitization in Comothers and PRL \\ Signalling in Mice}

In mice, pup-naïve virgin females display no avoidance for pups (see Fig. 3) and, consequently, exhibit qua- 
si-spontaneous maternal behaviour. However, full maternal behaviour in virgin female mice also requires a process of sensitization $[15,16]$ that leads to increased motivation for pups [15] (this work). One of the aims of this work was to understand whether maternal sensitization in virgin females influences PRL signalling in the brain. To do so, we used the comother model of maternal sensitization in which virgin females continuously interact with a dam (since postconception day 10) and her litter. This allows testing the relative role of stimuli provided by social interaction (mainly pup-derived stimulation) and of exposure to hormones during pregnancy and postpartum. Differences between comothers and dams are to be attributed to the effects of hormones associated to motherhood, whereas differences between comothers and virgins are likely due to the effects of social stimuli.

This strategy reveals that, as expected, hormones have a relevant influence on PRL signalling in most of the studied nuclei, including the CeM, MePD, LSV, Pa, LPAG, and PIL. This is mainly explained by the high PRL circulating levels observed in dams, as compared to virgin females (including comothers, see Fig. 7). In some of these nuclei, however, pSTAT5-ir cell density does not differ significantly between comothers and dams, namely, the LSV, AC/ADP, and MPO. This suggests that social stimuli might have an influence on PRL signalling at this level. In the case of the MPO, in addition, comothers show a significantly higher density of pSTAT5-ir cells than pupnaïve virgins. Therefore, our data provide evidence that maternal sensitization involves changes in PRL signalling, at least in the MPO, and maybe also in a specific subset of its afferents (LSV [68] and PAG [69]). Globally, this reinforces the view that the MPO occupies a central position in a brain circuit in which the responsiveness to PRL seems critical for the increased motivation for pups in dams and, also, in sensitized virgin females.

Since increase in PRL signalling observed in comothers is restricted to a few specific regions of the brain, it is not likely that it is due to increased levels of circulating PRL. In fact, the results of Experiment 2 indicate that serum PRL levels are similar in virgins and comothers, and significantly lower than those observed in dams (Fig. 7). Therefore, the observed effect on pSTAT5-ir is not a consequence of increased amounts of PRL accessing the brain from blood, but rather to a specific and localized increase in the sensitivity or responsiveness to the hormone.

Two mechanisms might explain such an increase in responsiveness to PRL. First, it can be due to an up-regulation of PRL receptors or other proteins of the Jak/STAT signalling cascade [70]. In the same way as expression of the PRL receptor increases in female mice during specific phases of the oestrous cycle, during pregnancy $[71,72]$ or specifically in the medial preoptic region after reproductive experience [73], a similar increase might occur in comothers, triggered by sustained exposure to pups (and/or to a pregnant female). This effect would require that pupderived stimuli reach the MPO and some of its afferents, where they may potentiate PRL signalling by modifying expression of PRL receptors or of elements of their signalling cascade. As discussed above, the pathway from the PIL to the MPO conveys somatosensory information of the ventral trunk (including suckling stimulation [55]), thus allowing such an effect of pup-derived somatosensory stimuli. Apparently, comothers have not developed nipples, and they have PRL levels similar to pup-naïve virgins. In addition, other models of maternal sensitization in mice [15] also resulting in increased motivation for pups are successful even in ovariectomized virgin females. These data suggest that nipple stimulation has a minor role in maternal sensitization in mice, whereas other kinds of somatosensory stimulation plus pup-derived chemosensory stimuli converging in the MePD (which receives inputs from both, main and accessory olfactory nuclei [57]) might also be involved in the pupinduced changes in PRL signalling observed in the MPO of comothers.

A second, alternative explanation of the increase in pSTAT5-ir cell density observed in the brain of comothers would be that pup-stimuli could induce local secretion of PRL or lactogens within the MPO, rather than changes in its signalling efficiency. This will be discussed below.

\section{Patterns of Brain PRL Signalling Related to}

\section{Differences in Maternal Motivation}

It is generally assumed that PRL influence on behaviour is mediated by hypophyseal PRL or placental lactogens crossing the blood-brain-barrier by means of its active transport by the choroid plexuses probably mediated by PRL receptor [74] (but see [75]). Consequently, the intracerebral levels of PRL are correlated with the concentration of PRL in the CSF $[76,77]$. Therefore, one would expect a positive correlation between the levels of pSTAT5-ir cell density in most of the nuclei of the brain analysed. This, however, is only happening in virgin females, where pSTAT5-ir cell density is correlated in most cases (see Table 2A), whereas the number of correlations is much lower in comothers and very low in dams.

A site-specific change in pSTAT5-ir cell density was already observed in lactating mice dams [32] and this was 
interpreted as due to local changes in the efficiency of PRL signalling. However, our finding does not easily fit a simple change in the efficiency of PRL signalling in some nuclei of dams and comothers. An increase or a decrease in the expression of PRL receptors or of the elements of its signalling cascade in a given nucleus would induce a change in the density of pSTAT5-ir cells in the nucleus, but the resulting density would still reflect the concentration of PRL reaching the nucleus. If this concentration is simply dependent on the circulating level of PRL and its transport into the brain through the choroid plexuses (as suggested by studies in primates and humans $[76,77]$ ), the different nuclei would still show positive correlation in their density of pSTAT5-ir.

The most likely explanation for this loss of correlation between observed PRL signalling in the different nuclei is that local sources of PRL come into play during motherhood and, to a lesser extent, with pup-sensitization. Thus, PRL mRNA has been detected in the SO and $\mathrm{Pa}$ of the brain of rats [78], and PRL protein is present in several areas of the hypothalamus of the rat [79]. In addition, PRL-like immunoreactive neurons are observed in the SO and $\mathrm{Pa}$, and immunoreactive fibres innervate portions of the $\mathrm{Pa}$, amygdala [80], and preoptic region. A similar pattern of immunoreactivity has recently been confirmed in the mouse brain by our group [81]. These data indicate that some neurons express and likely secrete PRL from axon terminals in specific sites of the brain, as demonstrated in the Pa and MPO of rats [82]. Interestingly, lactation is accompanied by an increase in the expression of PRL in the brain of female rats [83].

Changes in the production and/or synaptic release of PRL induced during pregnancy and postpartum (dams) or interaction with pups during maternal sensitization (comothers) might also explain the increase in PRL signalling observed in the MPO and PIL dams and comothers as compared to pup-naïve virgin females. Given the critical role of PRL signalling in the MPO on the establishment of maternal behaviour [52], these changes might have a mechanistic role on the establishment of maternal motivation for pups in both dams and comothers. This idea does not fit, however, the lack of pSTAT5-ir cells observed in lactating mouse dams after bromocriptine injections blocking hypophysial PRL release [32]. Further experiments specifically addressing this issue should be performed to explore whether neural PRL, probably released in specific synapses in response to specific stimuli (e.g., pup-derived stimuli) might have a role in the induction of motivated maternal behaviour.

Neuroendocrinology of Maternal Motivation

\section{Conclusions}

Summarizing, we have developed and validated a novel test for maternal motivation based on the pup retrieval test and the effort-based decision-making paradigm. This design has revealed clear differences in maternal motivation among dams, sensitized virgin females (comothers), and pup-naïve virgins. According to this, although sensitized virgins apparently display full maternal behaviour [16], some aspects of maternal behaviour such as maternal aggression [14, 19] and high maternal motivation (data in this work) are only observed in dams, indicating that endocrine signals of pregnancy are necessary for their development. However, in comothers, continuous pup exposure leads to a moderate increase in motivation for pups. In addition, comothers display increased responsiveness to PRL in the MPO and PIL, which belong, together with the medial amygdala, to a circuit involved in transmitting and integrating pup-derived stimuli to facilitate proactive (motivated) maternal responses. Altogether, this evidence supports the hypothesis that stimuli derived from pups (and a pregnant/postpartum female), selectively increase PRL responsiveness in discrete brain sites, enabling a priming action of the hormones to increase motivation for pups, and facilitate proactive maternal responses. This coordinated action of pup-derived stimuli (maybe also stimuli derived from the accompanying dam) and altered PRL signalling constitutes a putative mechanism underlying maternal sensitization. Our correlation data also provide indirect evidence suggestive of a possible role of brain-secreted PRL in maternal motivation, and specifically in increased maternal sensitization in comothers.

\section{Acknowledgements}

The authors are indebted to Dr. Mercè Correa for the enriching discussion on the design of the MPRT and to Dr. M. Victoria Ibañez-Gual (associate professor of statistics) for her help with the analysis of the data on pSTAT5-ir cell density. We also thank Dr. Manuela Barneo-Muñoz and Cinta Navarro-Moreno for their help and support during the experimental part of the work and the analysis of data.

\section{Statement of Ethics}

Animals were treated throughout according to the European Union Council Directive of June 3, 2010 (6106/1/10 REV1). Accordingly, procedures were approved by the Committee of Ethics on Animal Experimentation of the Jaume I University of Castellón

Neuroendocrinology 2021;111:805-830 
(UJI), where the experiments were performed on behalf of the "Conselleria de Presidencia y Agricultura, Pesca, Alimentación y Agua" of the Valencian Government. Approval number: 2015/ VSC/PEA/00055.

\section{Conflict of Interest Statement}

The authors have no conflicts of interest to declare.

\section{Funding Sources}

F.M.-G., C.-A.-P., and E.L. were funded by the Spanish Ministry of Economy and Competitiveness-FEDER and Ministry of Science and Innovation (BFU2016-77691-C2-2-P and C2-1-P; PID2019107322GB-C21 and-C22). F.M.-G., C.A.-P., and E.L. were funded by the Generalitat Valenciana (PROMETEO/2016/076). F.M.-G. was funded by the Universitat Jaume I de Castelló (UJI-B2016-45). H.S.-L. has been a predoctoral fellow of the FPU (Formación de Profesorado Universitario) programme of the Spanish Ministry of Education, Culture and Sport. The funders had no role in study design, data collection and analysis, decision to publish, or preparation of the manuscript.

\section{Author Contributions}

H.S.-L., F.M.-G., and C.A.-P. designed the study. F. M.-G., E.L., and C.A.-P. obtained funding and supervised the study. H.S.L. performed most of the experiments, in collaboration with M. A.-A. and M.B. for some of the experimental procedures. H.S.-L. and F.M.-G. analysed the material, obtained the data, and prepared the manuscript draft. All 6 authors revised critically the manuscript, a process in which they contributed with important intellectual content to it. Finally, all 6 authors approved the version to be submitted and (eventually) published.

\section{References}

1 Numan M, Insel TR. The neurobiology of parental behavior. New York: Springer-Verlag, 2003.

2 Rutter M, Kumsta R, Schlotz W, SonugaBarke E. Longitudinal studies using a "natural experiment" design: the case of adoptees from Romanian institutions. J Am Acad Child Adolesc Psychiatry. 2012 Aug;51(8):762-70.

3 Anacker C, O’Donnell KJ, Meaney MJ. Early life adversity and the epigenetic programming of hypothalamic-pituitary-adrenal function. Dialogues Clin Neurosci. 2014 Sep; 16(3):321-33.

4 Mehta MA, Golembo NI, Nosarti C, Colvert E, Mota A, Williams SC, et al. Amygdala, hippocampal and corpus callosum size following severe early institutional deprivation: the English and Romanian adoptees study pilot. J Child Psychol Psychiatry. 2009 Aug;50(8) 943-51.

5 Gammie SC. Current models and future directions for understanding the neural circuitries of maternal behaviors in rodents. Behav Cogn Neurosci Rev. 2005;4(2):119-35.

6 Numan M, Stolzenberg DS. Hypothalamic interaction with the mesolimbic dopamine system and the regulation of maternal responsiveness. Neurobiol Parental Brain. 2008:322.

7 Lee A, Clancy S, Fleming AS. Mother rats barpress for pups: effects of lesions of the mpoa and limbic sites on maternal behavior and operant responding for pup-reinforcement. Behav Brain Res. 2000 Mar;108(2):215-31.

8 Pereira M, Morrell JI. Functional mapping of the neural circuitry of rat maternal motivation: effects of site-specific transient neural inactivation. J Neuroendocrinol. 2011 Nov; 23(11):1020-35.
9 Olazábal DE, Pereira M, Agrati D, Ferreira A, Fleming AS, González-Mariscal G, et al. New theoretical and experimental approaches on maternal motivation in mammals. Neurosci Biobehav Rev. 2013;37(8):1860-74.

10 Bridges RS. Neuroendocrine regulation of maternal behavior. Front Neuroendocrinol. 2014;36:178-96.

11 Numan M, Insel TR. The neurobiology of parental behavior. New York: Springer-Verlag; 2003.

12 Seip KM, Morrell JI. Exposure to pups influences the strength of maternal motivation in virgin female rats. Physiol Behav. 2008 Nov; 95(4):599-608.

13 Bridges RS, DiBiase R, Loundes DD, Doherty PC. Prolactin stimulation of maternal behavior in female rats. Science. 1985 Feb;227(4688): $782-4$.

14 Martín-Sánchez A, Valera-Marín G, Hernández-Martínez A, Lanuza E, Martínez-García F, Agustín-Pavón C. Wired for motherhood: induction of maternal care but not maternal aggression in virgin female CD1 mice. Front Behav Neurosci. 2015 Jan;9:197.

15 Stolzenberg DS, Rissman EF. Oestrogen-independent, experience-induced maternal behaviour in female mice. J Neuroendocrinol. 2011;23(4):345-54.

16 Alsina-Llanes M, De Brun V, Olazábal DE. Development and expression of maternal behavior in naïve female C57BL/6 mice. Dev Psychobiol. 2015;57(2):189-200.

17 Yohn SE, Collins SL, Contreras-Mora HM, Errante EL, Rowland MA, Correa M, et al. Not all antidepressants are created equal: differential effects of monoamine uptake inhibitors on effort-related choice behavior. Neuropsychopharmacology. 2016 Feb;41(3):68694.
18 Cousins MS, Atherton A, Turner L, Salamone JD. Nucleus accumbens dopamine depletions alter relative response allocation in a T-maze cost/benefit task. Behav Brain Res. 1996; 74(1-2):189-97.

19 Martín-Sánchez A, McLean L, Beynon RJ Hurst JL, Ayala G, Lanuza E, et al. From sexual attraction to maternal aggression: when pheromones change their behavioural significance. Horm Behav. 2015 Feb;68:65-76.

20 Terkel J, JS. Humoral factors underlying maternal behavior at parturition: corss transfusion between freely moving rats. J Comp Physiol Psychol. 1972;80(3):365-71.

21 Bridges RS, Ronsheim PM. Prolactin (PRL) regulation of maternal behavior in rats: bromocriptine treatment delays and PRL promotes the rapid onset of behavior. Endocrinology. 1990 Feb;126(2):837-48.

22 Soares MJ. The prolactin and growth hormone families: pregnancy-specific hormones/cytokines at the maternal-fetal interface. Reprod Biol Endocrinol. 2004 Jul;2:51.

23 Bridges RS. The role of lactogenic hormones in maternal behavior in female rats. Acta Paediatr Suppl. 1994 Jun;397(Suppl 1):33-9.

24 Numan M, Woodside B. Maternity: neural mechanisms, motivational processes, and physiological adaptations. Behav Neurosci. 2010;124(6):715-41.

25 Brown RS, Kokay IC, Herbison AE, Grattan DR. Distribution of prolactin-responsive neurons in the mouse forebrain. J Comp Neurol. 2010;518(1):92-102.

26 Salais-López H. Mapping the actions of prolactin in the mouse brain. Sexual dimorphism, steroid regulation and the neuroendocrinology of maternal behaviour. València, Spain: Universitat de València; 2017. Available from: http://hdl.handle.net/10550/59359. 
27 Salais-López H, Agustín-Pavón C, Lanuza E, Martínez-García F. The maternal hormone in the male brain: sexually dimorphic distribution of prolactin signalling in the mouse brain. PLoS One. 2018;13(12):e0208960.

28 Maestripieri D, Alleva E. Litter defence and parental investment allocation in house mice. Behav Processes. 1991 May;23(3):223-30.

29 Kohl J, Babayan BM, Rubinstein ND, Autry AE, Marin-Rodriguez B, Kapoor V, et al. Functional circuit architecture underlying parental behaviour. Nature. 2018;556(7701): $326-31$.

30 Salais-López H, Lanuza E, Agustín-Pavón C, Martínez-García F. Tuning the brain for motherhood: prolactin-like central signalling in virgin, pregnant, and lactating female mice. Brain Struct Funct. 2017;222(2):895-921.

31 Salais-López H, Agustín-Pavón C, Lanuza E, Martínez-García F. The maternal hormone in the male brain: sexually dimorphic distribution of prolactin signalling in the mouse brain. PLoS One. 2018;13(12):e0208960.

32 Brown RS, Herbison AE, Grattan DR. Differential changes in responses of hypothalamic and brainstem neuronal populations to prolactin during lactation in the mouse. Biol Reprod. 2011;84(4):826-36.

33 Paxinos G, Franklin KBJ. The mouse brain in stereotaxic coordinates. San Diego, CA: Elsevier Academic Press; 2004.

34 Saltzman W, Ziegler TE. Functional significance of hormonal changes in mammalian $\mathrm{fa}$ thers. J Neuroendocrinol. 2014;26(10):68596.

35 Armario A, Lopez-Calderón A, Jolin T, Castellanos JM. Sensitivity of anterior pituitary hormones to graded levels of psychological stress. Life Sci. 1986 Aug;39(5):471-5.

36 Borrell J, Piva F, Martini L. Effect of pentobarbital on serum levels of LH, FSH and prolactin in long-term ovariectomized rats. Neuroendocrinology. 1978;27(5-6):239-46.

37 Salamone JD, Yohn SE, López-Cruz L, San Miguel N, Correa M. Activational and effortrelated aspects of motivation: neural mechanisms and implications for psychopathology. Brain. 2016 May;139(Pt 5):1325-47.

38 Bridges R, Zarrow MX, Gandelman R, Denenberg VH. Differences in maternal responsiveness between lactating and sensitized rats. Dev Psychobiol. 1972;5(2):123-7.

39 Mayer AD, Freeman NC, Rosenblatt JS. Ontogeny of maternal behavior in the laboratory rat: factors underlying changes in responsiveness from 30 to 90 days. Dev Psychobiol. 1979 Sep;12(5):425-39.

40 Mackinnon DA, Stern JM. Pregnancy duration and fetal number: effects on maternal behavior in rats. Physiol Behav. 1977 May; 18(5): 793-7.

41 Stern JM, Mackinnon DA. Postpartum, hormonal, and nonhormonal induction of maternal behavior in rats: effects on T-maze retrieval of pups. Horm Behav. 1976 Sep;7(3): 305-16.
42 Neumann ID, Torner L, Wigger A. Brain oxytocin: differential inhibition of neuroendocrine stress responses and anxiety-related behaviour in virgin, pregnant and lactating rats. Neuroscience. 2000 Jan;95(2):567-75.

43 Carratalá-Ros C, López-Cruz L, SanMiguel N, Ibáñez-Marín P, Martínez-Verdú A, Salamone JD, et al. Preference for exercise vs. More sedentary reinforcers: validation of an animal model of tetrabenazine-induced anergia. Front Behav Neurosci. 2020 Jan;13:1-15.

44 Crawley J, Bailey K. Anxiety-related behaviors in mice. In: Buccafusco JJ, editor. Meth ods of behavour analysis in neuroscience. 2nd ed. Boca Raton, FL: CRC Press/Taylor \& Francis; 2008. p. 77-101.

45 Bales KL, Saltzman W. Fathering in rodents: neurobiological substrates and consequences for offspring. Horm Behav. 2016;77:249-59.

46 Newman SW. The medial extended amygdala in male reproductive behavior. A node in the mammalian social behavior network. Ann N Y Acad Sci. 1999;877:242-57.

47 Kokay IC, Wyatt A, Phillipps HR, Aoki M, Ectors F, Boehm U, et al. Analysis of prolactin receptor expression in the murine brain using a novel prolactin receptor reporter mouse. J Neuroendocrinol. 2018;30(9):1-20.

48 Goudreau JL, Lindley SE, Lookingland KJ, Moore KE. Evidence that hypothalamic periventricular dopamine neurons innervate the intermediate lobe of the rat pituitary. Neuroendocrinology. 1992 Jul;56(1):100-5.

49 DeMaria JE, Lerant AA, Freeman ME. Prolactin activates all three populations of hypothalamic neuroendocrine dopaminergic neurons in ovariectomized rats. Brain Res. 1999 Aug; 837(1-2):236-41.

50 Anderson ST, Barclay JL, Fanning KJ, Kusters DH, Waters MJ, Curlewis JD. Mechanisms underlying the diminished sensitivity to prolactin negative feedback during lactation: reduced STAT5 signaling and up-regulation of cytokine-inducible SH2 domain-containing protein (CIS) expression in tuberoinfundibular dopaminergic neurons. Endocrinology. 2006;147(3):1195-202.

51 Bridges RS, Numan M, Ronsheim PM, Mann $\mathrm{PE}$, Lupini CE. Central prolactin infusions stimulate maternal behavior in steroid-treated, nulliparous female rats. Proc Natl Acad Sci U S A. 1990;87(20):8003-7.

52 Brown RSE, Aoki M, Ladyman SR, Phillipps HR, Wyatt A, Boehm U, et al. Prolactin action in the medial preoptic area is necessary for postpartum maternal nursing behavior. Proc Natl Acad Sci U S A. 2017;114(40):10779-84.

53 Menon R, Grund T, Zoicas I, Althammer F, Fiedler D, Biermeier V, et al. Oxytocin signaling in the lateral septum prevents social fear during lactation. Curr Biol. 2018;28(7):106678.e6.
54 Cservenák M, Bodnár I, Usdin TB, Palkovits M, Nagy GM, Dobolyi A. Tuberoinfundibular peptide of 39 residues is activated during lactation and participates in the suckling-induced prolactin release in rat. Endocrinology. 2010;151(12):5830-40

55 Cservenák M, Szabó ÉR, Bodnár I, Lékó A, Palkovits M, Nagy GM, et al. Thalamic neuropeptide mediating the effects of nursing on lactation and maternal motivation. Psychoneuroendocrinology. 2013 Dec;38(12):3070-84.

56 Pardo-Bellver C, Cádiz-Moretti B, Novejarque A, Martínez-García F, Lanuza E. Differential efferent projections of the anterior, posteroventral, and posterodorsal subdivisions of the medial amygdala in mice. Front Neuroanat. 2012 Aug;6:33-26.

57 Cádiz-Moretti B, Otero-García M, MartínezGarcía F, Lanuza E. Afferent projections to the different medial amygdala subdivisions: a retrograde tracing study in the mouse. Brain Struct Funct. 2016;221(2):1033-65.

58 Kohl J, Dulac C. Neural control of parental behaviors. Curr Opin Neurobiol. 2018 Apr; 49:116-22.

59 Lin R, Li Y, Luo M. A neural circuit driving maternal behaviors. Neuron. 2018 Apr;98(1): 6-8.

60 Bridges RS, Freemark MS. Human placental lactogen infusions into the medial preoptic area stimulate maternal behavior in steroidprimed, nulliparous female rats. Horm Behav. 1995 Jun;29(2):216-26.

61 Bridges RS, Numan M, Ronsheim PM, Mann $\mathrm{PE}$, Lupini CE. Central prolactin infusions stimulate maternal behavior in steroid-treated, nulliparous female rats. Proc Natl Acad Sci U S A. 1990 Oct;87(20):8003-7.

62 Brown RSE, Aoki M, Ladyman SR, Phillipps HR, Wyatt A, Boehm U, et al. Prolactin action in the medial preoptic area is necessary for postpartum maternal nursing behavior. Proc Natl Acad Sci U S A. 2017;114(40):10779-84.

63 Knobloch HS, Charlet A, Hoffmann LC, Eliava $\mathrm{M}$, Khrulev S, Cetin AH, et al. Evoked axonal oxytocin release in the central amygdala attenuates fear response. Neuron. 2012;73(3): $553-66$.

64 Neumann ID, Wigger A, Krömer S, Frank E, Landgraf R, Bosch OJ. Differential effects of periodic maternal separation on adult stress coping in a rat model of extremes in trait anxiety. Neuroscience. 2005;132(3):867-77.

65 Bosch OJ. Maternal nurturing is dependent on her innate anxiety: the behavioral roles of brain oxytocin and vasopressin. Horm Behav. 2011 Feb;59(2):202-12.

66 Kessler MS, Bosch OJ, Bunck M, Landgraf R, Neumann ID. Maternal care differs in mice bred for high vs. low trait anxiety: impact of brain vasopressin and cross-fostering. Soc Neurosci. 2011 Jan;6(2):156-68.

67 Fairbanks LA. Individual differences in maternal style causes and consequences for mothers and offspring. Adv Study Behav. 1996;25:579-611. 
68 Risold PY, Swanson LW. Connections of the rat lateral septal complex. Brain Res Brain Res Rev. 1997 Sep;24(2-3):115-95.

69 Rizvi TA, Ennis M, Shipley MT. Reciprocal connections between the medial preoptic area and the midbrain periaqueductal gray in the rat: a WGA-HRP and PHA-L study. J Comp Neurol. 1992;315:1-15.

70 Freeman ME, Kanyicska B, Lerant A, Nagy G. Prolactin: structure, function, and regulation of secretion. Physiol Rev. 2000 Oct; 80(4): 1523-631.

71 Sugiyama T, Minoura H, Kawabe N, Tanaka M, Nakashima K. Preferential expression of long form prolactin receptor mRNA in the rat brain during the oestrous cycle, pregnancy and lactation: hormones involved in its gene expression. J Endocrinol. 1994 May;141(2): 325-33.

72 Bridges RS, Hays LE. Steroid-induced alterations in mRNA expression of the long form of the prolactin receptor in the medial preoptic area of female rats: effects of exposure to a pregnancy-like regimen of progesterone and estradiol. Brain Res Mol Brain Res. 2005; 140(1-2):10-6.
73 Anderson GM, Grattan DR, Van Den Ancker $\mathrm{W}$, Bridges RS. Reproductive experience increases prolactin responsiveness in the medial preoptic area and arcuate nucleus of female rats. Endocrinology. 2006;147(10):4688-94.

74 Mangurian LP, Walsh RJ, Posner BI. Prolactin enhancement of its own uptake at the choroid plexus. Endocrinology. 1992 Aug;131(2): 698-702.

75 Brown RS, Wyatt AK, Herbison RE, Knowles PJ, Ladyman SR, Binart N, et al. Prolactin transport into mouse brain is independent of prolactin receptor. FASEB J. 2016;30(2): 1002-10.

76 Martensz ND, Herbert J. Relationship between prolactin in the serum and cerebrospinal fluid of ovariectomized female rhesus monkeys. Neuroscience. 1982;7(11):2801-12.

77 Markianos M, Koutsis G, Evangelopoulos ME, Mandellos D, Sfagos C. Serum and cerebrospinal fluid prolactin levels in male and female patients with clinically-isolated syndrome or relapsing-remitting multiple sclerosis. J Neuroendocrinol. 2010;22(6):503-8.

78 Emanuele NV, Jurgens JK, Halloran MM, Tentler JJ, Lawrence AM, Kelley MR. The rat prolactin gene is expressed in brain tissue: detection of normal and alternatively spliced prolactin messenger RNA. Mol Endocrinol. 1992;6(1):35-42.
79 Mejía S, Morales MA, Zetina ME, Martínez de la Escalera G, Clapp C. Immunoreactive prolactin forms colocalize with vasopressin in neurons of the hypothalamic paraventricular and supraoptic nuclei. Neuroendocrinology. 1997 Sep;66(3):151-9.

80 Paut-Pagano L, Roky R, Valatx JL, Kitahama $\mathrm{K}$, Jouvet M. Anatomical distribution of prolactin-like immunoreactivity in the rat brain. Neuroendocrinology. 1993 Dec;58(6):68295.

81 Abellan-Álvaro M. The medial amygdala as a key neural centre in maternal aggression: genetic, neural and behavioural analysis. València, Spain: Universitat de València; 2020. Available from: https://www.educacion.es/ teseo/mostrarRef.do?ref=1837302.

82 Torner L, Maloumby R, Nava G, Aranda J, Clapp C, Neumann ID. In vivo release and gene upregulation of brain prolactin in response to physiological stimuli. Eur J Neurosci. 2004;19(6):1601-8.

83 Torner L, Toschi N, Nava G, Clapp C, Neumann ID. Increased hypothalamic expression of prolactin in lactation: involvement in behavioural and neuroendocrine stress responses. Eur J Neurosci. 2002;15(8):1381-9. 